\title{
Perceptual Organisation Affects Perception and Appreciation of Abstract Art: A Case Study with Black Square and Red Square by Kazimir Malevich
}

\author{
Arefe Sarami $^{1, *}$, Reza Afhami ${ }^{1, * *, * * *}$ and Johan Wagemans ${ }^{2, * * * *}$ \\ ${ }^{1}$ Department of Art and Architecture, Tarbiat Modares University, Al Ahmad Street, \\ 14115-111 Tehran, Iran \\ ${ }^{2}$ Department of Brain \& Cognition, University of Leuven (KU Leuven), Tiensestraat \\ 102 - box 3711, 3000 Leuven, Belgium
}

Received 5 March 2021; accepted 21 September 2021

\begin{abstract}
Perceptual organisation is hypothesised as a key in the perception and appreciation of abstract art. Here, we investigated how relational and compositional features affected the perception and aesthetic appreciation of Black Square and Red Square by Kazimir Malevich (1915). We studied how (i) the presence and obliquity of the red square and (ii) the relative configuration of the black and red square affected the detectability of the obliquity of the black square in this artwork. Results showed that the simultaneous presence and obliquity of the red square masked the obliquity of the original black square. The likelihood of the black square being incorrectly perceived as an exact square was always maximum in the original configuration and even slight alterations in the original configuration of the work resulted in the obliquity of the black square to be noticed. The original artwork was more aes-thetically preferred compared to its alternatives. We argue that the artist may have intentionally set the configuration to mask the obliquity of the black square and maximise the aesthetic preference.
\end{abstract}

\section{Keywords}

Visual art perception, perceptual organisation, shape perception, regularity, composition, abstract art, art experience, grouping principles

\footnotetext{
ORCID: 0000-0001-6332-5548

** To whom correspondence should be addressed. E-mail: afhami@modares.ac.ir

*** ORCID: 0000-0002-7678-6164

**** ORCID: 0000-0002-7970-1541
} 


\section{General Introduction}

Black Square and Red Square by Kazimir Malevich (Fig. 1, left) is one of the canonical pieces of the art movement of Suprematism. In addition to its arthistorical significance (sketched further below), this painting has a large num-ber of characteristics that make it well suited to study the role of perceptual organisation in the perception and appreciation of abstract art. It has a kind of minimal composition, with two squares in a specific relation to one another, in terms of their size, colour, position, and orientation (see Fig. 1, right and

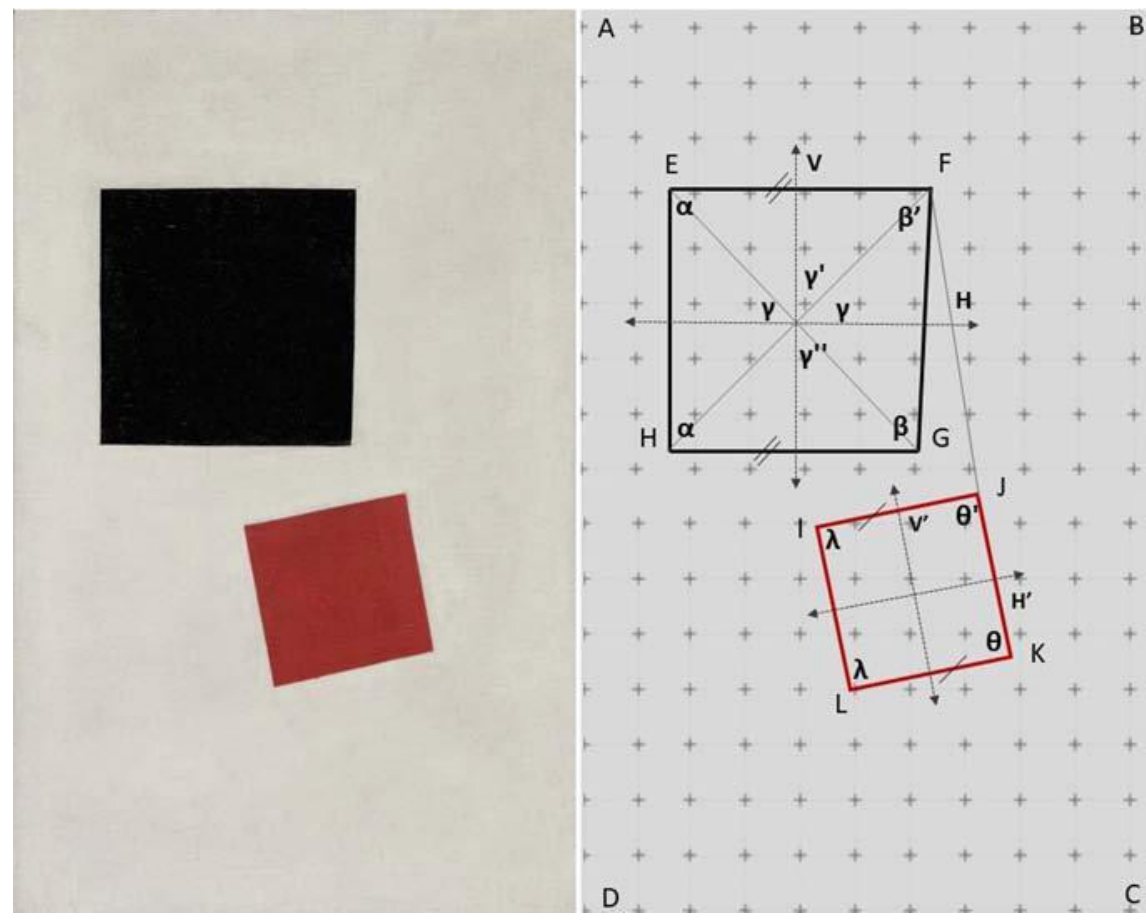

Figure 1. (Left) Black Square and Red Square (1915) by Kazimir Malevich and exhibited at ' 0,10 ', Petrograd. Secondary name: Painterly Realism of a Boy with Knapsack - Color Masses in the Fourth Dimension. The original work is oil on canvas $(71.12 \times 44.45 \mathrm{~cm})$ and is kept at the Museum of Modern Art, New York. (Right) Structure of the original work: V and H are the vertical and horizontal axes of the global reference system, respectively; V' and H' are the vertical and horizontal axes of the reference system aligned to the small red square, respectively. The relative angle between the two squares is $11^{\circ}$. The orientation of the right edge of the small red square deviates slightly from V' (see also Table 1 for more detailed information about the geometry of the painting). 
Table 1 for detailed geometric information). And yet, we believe this composition and these relations constitute major factors in how the artist conveyed the principles of Suprematism, and how this artwork is perceived and appreciated by its audience. Its minimal nature lends itself nicely for a systematic inves-tigation of the perceptual and aesthetic effects of the relational and composi-tional features of the painting. Most notably, the black square is not actually a perfect geometrical square (its right side is skewed, i.e., it does not form $90^{\circ}$ angles with the top and bottom sides; Douglas, 1998; Milner, 1996; see Note 1), but this distortion generally goes unnoticed by being embedded in a composition with a particularly coloured, positioned, and oriented red square

\section{Table 1.}

Black Square and Red Square: General and geometric information about the painting.

\begin{tabular}{ll}
\hline Canvas measures & $\begin{array}{l}\mathbf{7 1 . 1} \times \mathbf{4 4 . 5} \mathbf{~ c m ~ ( 2 8} \\
\times \mathbf{1 7} 1 / 2 \text { inches) }\end{array}$ \\
\hline Aspect ratio & $8: 5$ \\
Distance between two & $d\left(\mathrm{~V}, \mathrm{~V}^{\prime}\right)=9.6 \mathrm{~cm}$ \\
vertical axes & \\
Black square & $19.5 \mathrm{~cm}$ \\
Edge measures & $\alpha=90^{\circ}$ \\
Angles & $\beta=92^{\circ}$ \\
Angles & $\beta^{\prime}=88^{\circ}$ \\
& $\gamma=90^{\circ}$ \\
& $\gamma^{\prime}=91^{\circ}$ \\
& $\gamma^{\prime \prime}=89^{\circ}$ \\
& $7.25 \mathrm{~cm}$ \\
$d($ AC, EH) & $37.4 \mathrm{~cm}$ \\
$d$ (HG, DC) & $14.5 \mathrm{~cm}$ \\
Red Square & $\lambda=90^{\circ}$ \\
Edge measures & \\
Angles & \\
(angles of the red square & \\
diagonals $\approx 90)$ & $\theta=91^{\circ}$ \\
Angles & \\
(angles of the red square & $\theta^{\prime}=89^{\circ}$ \\
diagonals $\approx 90)$ & \\
Angles between two & $\mathrm{HH}=11^{\circ}$ \\
horizontal axes $(\mathrm{HH})$ & $31.0 \mathrm{~cm}$ \\
$d(\mathrm{IJ}$, DC) & $13.6 \mathrm{~cm}$ \\
$d($ JK, BC) & \\
\hline
\end{tabular}


(Crone and Moos, 1991; Milner, 1996). As we will demonstrate below, the extent to which the distortion is perceived or not strongly depends on the relations between the two squares and on the overall composition they form.

In Experiment 1, we focus on the perception of the black square as perfect or imperfect (distorted), as a function of the presence of the red square and the geometric properties of the two shapes. After having determined the main effect of the composition, Experiment 2 zooms in on the more specific contri-butions of the different factors underlying the composition, by parametrically varying the skewness of the right side of the black square, the proximity of the two squares, and the orientation of the red square, as well as by repeating the whole set of manipulations with a black version of the small square. Finally, Experiment 3 investigates how balanced, harmonious, pleasing and interesting the original composition and all of its variations are perceived to be.

All in all, we are convinced that this empirical study of the perception and appreciation of Black Square and Red Square constitutes a nice case study of the importance of the spatial relations between distinct, geometric components of an abstract painting and the role of its overall composition, however minimal. More generally, it also illustrates how the joint study of art and perception can strengthen both fields. On the one hand, we empirically confirm some of the major principles of an important art movement by carefully analyzing the perceptual and aesthetic effects of one of its prototypical paintings. On the other hand, we are able to disentangle the perceptual factors that are combined in such an artwork, and pinpoint their role in perceptual organisation of geometric compositions, and eventually in the perception and aesthetic appreciation of an important artwork.

Before we describe the specific aims, methods, and results of each of the experiments, we provide some art-historical context (mainly for the perception audience), and we cover some background on perceptual organisation and composition in relation to empirical aesthetics (mainly for readers with an interest in art as such).

\subsection{Introduction to the Art-Historical Context}

\subsubsection{Black Square and Red Square, Kazimir Malevich, and Suprematism}

Black Square and Red Square (1915) by Kazimir Malevich was first exhibited at 0,10 (pronounced 'zero-ten'), in Petrograd (now St. Petersburg, Russia), from 19 December 1915 to 17 January 1916. The original work is oil on can-vas (71.12 $\times 44.45 \mathrm{~cm}$ ) and is kept at the Museum of Modern Art, New York, under its secondary name Painterly Realism of a Boy with Knapsack - Color Masses in the Fourth Dimension. The exhibition marked the official beginning of Suprematism, an abstract art form the name of which refers to the supremacy of pure artistic feeling as opposed to the visual depiction of existing objects. 


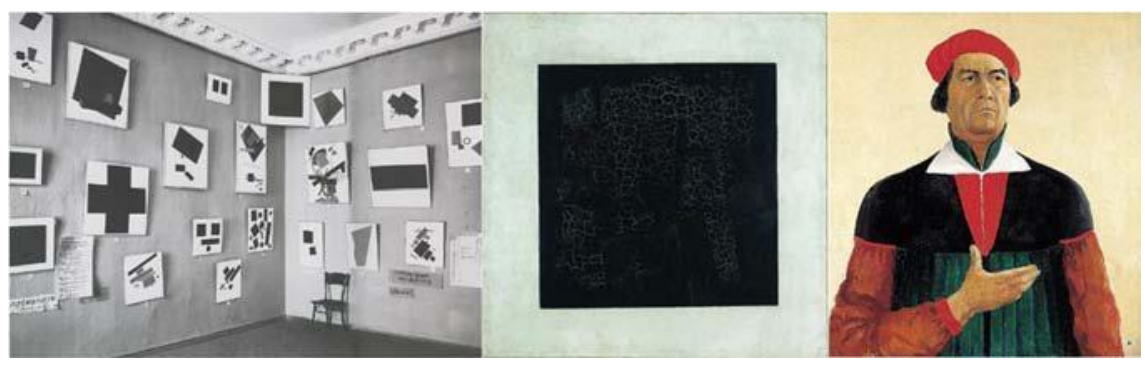

Figure 2. (Left) The only existing black-and-white photograph of the ' 0,10 ' exhibition. (Center) Black Square, 1915, oil on linen, $79.5 \times 79.5 \mathrm{~cm}$, Tretyakov Gallery, Moscow. (Right) Self-Portrait, 1933, oil on canvas, $73 \times 66 \mathrm{~cm}$, Russian State Museum, St. Petersburg.

This new art form replaced Cubo-Futurism, as marked by the full title of the exhibition: The Last Futurist Exhibition of Paintings 0,10. Cubo-Futurism was a Russian art movement, defined by an amalgamation of French Analytical Cubism and Italian Futurism, in the beginning of the 20th century.

The exhibition had 155 works on display, including 39 by Malevich, which occupied a central room (see Fig. 2, left). One of the most important pieces in Malevich's oeuvre, Black Square (1915, see Fig. 2, centre), was particularly noticeable and controversial because it was placed in a location where Russian Orthodox households place their icons (i.e., the top corner of the room). Malevich himself, and critics after him, often referred to Black Square as 'the zero point of painting', a real breakthrough in his career as well as in art more generally, because it constituted a new form of painting-pure form, emerging from nothing and depicting nothing. Two years before that, Malevich had already introduced the idea by using a black square as a stage curtain for a Futurist opera, and he repeatedly painted it afterwards (e.g., in 1923 and in 1929). He even included a reference to it in his famous self-portrait of 1933 , in a more traditional style of painting (because all forms of Modernism were by that time found bourgeois and banned by the Stalinist cultural policy and its focus on Social Realism), by holding his hand in a particular way and indicat-ing a virtual black-over-white square in the clothing (see Fig. 2, right). In fact, most of Malevich's paintings at the show, including Black Square and Red Square, contained some kind of distorted black square (see below for more details).

Kazimir Malevich (1879-1935) had initially worked in several different styles, such as Impressionism, Symbolism, Fauvism and Cubism, which he all quickly assimilated. Having exhibited in the famous Blaue Reiter exhibition of 1912 with Cubo-Futurist works, he was already an established painter when he developed the concept of Suprematism. In 1915, on the occasion of the 0,10 exhibition, Malevich published his manifesto, From Cubism to Suprematism, 
which constituted the foundations of the new movement. In 1927, Suprematism (Part II of his book The Non-Objective World) was published as Bauhaus Book No. 11 in Munich (translated into English in 1959). In this work, Malevich formulated the core concept of Suprematism in a concise and clear way: "Under Suprematism I understand the primacy of pure feeling in creative art. To the Suprematist, the visual phenomena of the objective world are, in them-selves, meaningless; the significant thing is feeling, as such, quite apart from the environment in which it is called forth" (p. 117).

Malevich's concept of Suprematism moved as far as possible away from the world of natural forms (objectivity) and sought to develop a form of expres-sion that provided access to "the supremacy of pure feeling" and spirituality. In order to achieve this, he created a Suprematist 'grammar' with basic geo-metric forms such as the square and the circle. Note, however, that oftentimes the forms in Malevich's work were not geometrically perfect. The intentional distortions, highly noticeable in Red Square (1915, see Fig. 3, left) and Hieratic Suprematist Cross (1921, see Fig. 3, centre), were introduced to induce appar-ent kinetic motion, imagined forms in movement or through time, often expressed in the primary (e.g., Dynamic Suprematism) or secondary titles associated with some of the works (e.g., Airplane Flying for Suprematist Composition). These secondary titles were also used ironically to express how the non-objectivity of Suprematism distinguishes itself from traditional repre-sentational art and painterly realism (e.g., Painterly Realism of a Peasant Woman in Two Dimensions for Red Square).

In Suprematist Composition: White on White (1918, see Fig. 3, right), Malevich superimposed a barely differentiated off-white square on an offwhite ground. Because this painting has lost most of the characteristics of

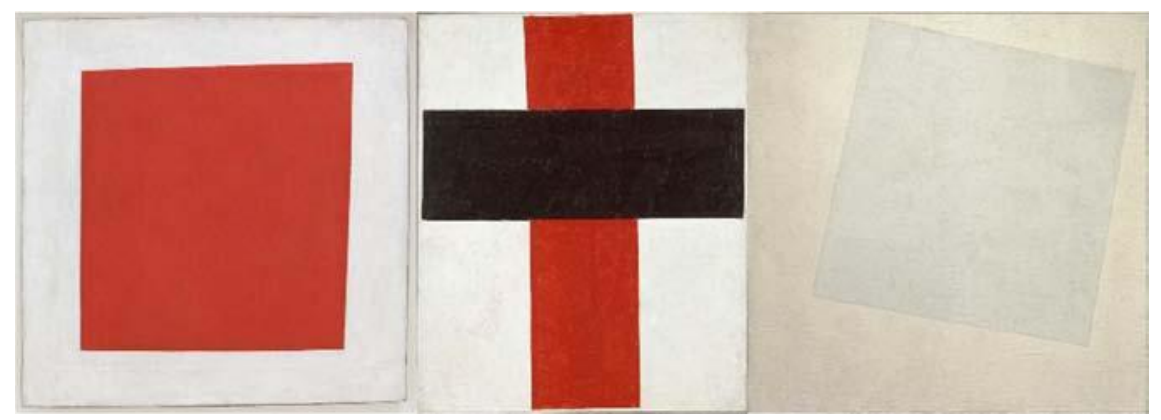

Figure 3. (Left) Red Square - Painterly Realism of a Peasant Woman in Two Dimensions, 1915, oil on canvas, $53 \times 53 \mathrm{~cm}$, Russian State Museum, St. Petersburg. (Center) Hieratic Suprematist Cross, 1921-1927?, oil on canvas, $84 \times 69.5 \mathrm{~cm}$, Stedelijk Museum, Amsterdam. (Right) Suprematist Composition - White on White, 1918, oil on canvas, $79.4 \times 79.4 \mathrm{~cm}$ Museum of Modern Art, New York. 
representational art, leaving only a simple monochrome geometrical shape, it seems to be the logical conclusion of Malevich's ideal of pure abstraction, with the colour white symbolising infinity. Nevertheless, in spite of the minimalism in shape, the slight tilt of the square suggests movement, with the tilted square perceived as coming out of the canvas strengthened by the slightly asymmetrical shape (painted with imprecisely defined boundaries and visible brush strokes).

Although Suprematism shares some similarities with Constructivism, they are also fundamentally different in two respects. First, Constructivism was characterised by a cult of the object, reflecting a utilitarian strategy of adapting art to the principles of functional organisation. In contrast, the philosophy behind Suprematism was profoundly anti-materialist and anti-utilitarian. In Malevich's own words (Suprematism, 1927, p. 118): “Art no longer cares to serve the state and religion, it no longer wishes to illustrate the history of manners, it wants to have nothing further to do with the object, as such, and believes that it can exist, in and for itself, without 'things' ". Second, Constructivism transformed the traditional easel painter into some kind of engineer, organis-ing life in all of its aspects and placing man at the centre of the universe. Instead, Suprematism regarded the artist as the originator and transmitter of the world's only true reality (i.e., absolute non-objectivity). In Malevich's own words (Suprematism, 1927, p. 118): “... a blissful sense of liberating non-objectivity drew me forth into a 'desert,' where nothing is real except feeling ...".

El Lissitzky, a contemporary painter who was strongly influenced by Suprematism but moved into Constructivism afterwards, was quite important in spreading Malevich's ideas and art form. During his trip to the West, on the occasion of the exhibition of Non-Objective Art in Berlin (1923), El Lissitzky was in close contact with Theo van Doesburg, forming a bridge between Suprematism and De Stijl. However, note that De Stijl (e.g., Piet Mondrian; see below) differs from Suprematism in fundamental ways as well, for instance, because of its exactly geometric compositions, with a number of white and coloured surfaces (in particular, red, yellow, and blue), nicely delineated by vertical and horizontal black lines of a given thickness, while Malevich painted more painterly, with more imperfections, more movement, more depth and gradually fewer shapes and less colour.

In addition to influencing his contemporaries, Malevich's art and writings also influenced later generations of abstract artists such as the Minimalists. Several of Malevich's paintings were included in the exhibition on Cubism and Abstract Art at the Museum of Modern Art in New York (1936). A few years later, Solomon R. Guggenheim, who was an early and passionate collec-tor of the Russian avantgarde, opened the Museum of Non-Objective Painting in New York (1939) because he was inspired by the same spiritual quest and 
aesthetic ideals as Malevich. Later, in 1973, the Guggenheim Museum held the first U.S. Malevich retrospective, followed, in 1989, by the first large-scale Malevich retrospective in Europe at the Stedelijk Museum in Amsterdam, which owns a substantial number of his art works. Both of these shows received much interest and caused a further increase of Malevich's impact on postwar American and European painters. Since then, the prices of his work have sky-rocketed as well. For instance, Black Square (i.e., the fourth version of his magnum opus painted in the 1920s) was sold in 2002 to the State Hermitage Museum in St. Petersburg for an equivalent of 1 million USD (financed by a Russian philanthropist). The Suprematist Composition from 1916 set the world record for any Russian work of art, when it was sold in 2008 at Sotheby's in New York for 60 million USD, 10 years later topped when it was sold at Christie's in New York for more than 85 million USD.

Several studies have been performed on Malevich's philosophical desires in his Suprematist works, his geometrical reasoning (Corrada, 1997; Galenson, 2008; Golding, 2000; Harrison and Wood, 2003; Luecking, 2010; Milner, 1996), and his choice of colours (Tao et al., 2014). To Malevich, geometry facilitated surpassing the physical world in art (Luecking, 2010). In an inter-esting monograph, Crone and Moos (1991) presented Malevich as a uniquely creative artist, embodying in his works many of the insights in theoretical physics and nonEuclidean geometry that are still widely misunderstood today. Although El Lissitzky called the state of space in Malevich's paintings 'irra-tional' (i.e., without any representational constructs), perspectival positions in space have been embodied by proportions and ratios in his work (Harrison and Wood, 2003). In fact, the paintings of Malevich were a new way of generating a dynamic world of geometric harmonies according to some art theorists (Kochman, 1999), producing a space without any horizon and yet still preserv-ing geometric relationships (Luecking, 2010). However, until today, the gen-eral role of geometric composition and the specific effects of the geometric configuration on the perception of its basic geometric forms in Malevich's works have not been empirically investigated yet, as far as we know.

In this study, we focused on one particular artwork presented in 0,10, the Black Square and Red Square, and investigated whether the obliquity of the black square in this work remains unnoticed due to the specific composition of the painting (i.e., the influence of the smaller, tilted red square in the lower right corner on the perception of the black square). The canvas measures 71.1

$\times 44.5 \mathrm{~cm}$ and thus has a ratio of $8: 5$, which is a rectangle with a golden section. Malevich hung this painting in different orientations (i.e., as in Fig. 1 or upside down) in different exhibitions. According to Milner's (1996) analysis of this painting (Note 2), the black square, which was displayed as a single form elsewhere in the same room at the 0,10 show, was shifted to the right in this painting, parallel to the global horizontal axis, and a tilted smaller red 
square was added in the right corner of the black square. As a result, the black square appears static and the red square dynamic, with its pungent red being equivalent in visual energy to the larger black square, while they are related both by shape and by position. The black square's edge measures $19.5 \mathrm{~cm}$. The diagonals of the black square form a cross at almost right angles in the central point (Fig. 1, right and Table 1). Although the skewness makes it appear slightly asymmetrical, it is actually perfectly symmetrical about the right diag-onal axis. According to some authors (Luecking, 2010; Milner, 1996), the skewness of the black square has an intense visual effect, as if the square has been distorted or its unbalanced form has begun to fall. The tilted red square's edge measures $14.5 \mathrm{~cm}$, is positioned below and to the right of the black square, and is also not an exact square, although its distortion is less than in the black square or similar shapes in other works by Malevich (see Fig. 3, left and centre). Note, however, that none of these observations by art critics on the perceptual and aesthetic effects of these geometric shape distortions and spa-tial relations has been tested in a larger sample of naïve or expert observers, to the best of our knowledge, which is what we set out to do in this study.

\subsection{Introduction to Perceptual Organisation and Composition in Relation} to Empirical Aesthetics

Perceptual organisation and empirical aesthetics are both broad, rich and active research domains. However, theoretical and empirical work which is focused specifically on the role of perceptual organisation in determining beauty, liking or interest of patterns, images and artworks is scarce and much less developed than it could be (for interesting exceptions, see Koenderink, 2015a, b; Muth et al., 2015; Spehar and van Tonder, 2017; Van de Cruys and Wagemans, 2011a, b; van Tonder and Spehar, 2013; Wagemans, 2017). Here, we briefly introduce each of the two broad domains and then focus in more depth on a number of issues that are directly relevant to how perceptual organisation could affect the perception and appreciation of Malevich's Black Square and Red Square.

Perceptual organisation, defined as the organisation of elements or features of patterns of light captured by the eye (whether reflected from objects in the real world or from pigments painted on a canvas) into groups or structures, is a crucial aspect of how the visual system makes sense of the input. Organised entities are an important component of our fluctuating visual experience and they are often also considered as a necessary step in the middle levels of the visual processing stream across the cortical hierarchy, in-between elementary, local attributes of stimulus patches captured by neurons with small receptive fields, on the one hand and integrated, visual and semantic interpretations of seemingly complete scenes or events, on the other hand. Perceptual 
organisation comprises a wide range of processes such as perceptual grouping and figure-ground organisation, for which Gestalt psychologists have speci-fied grouping principles and Gestalt laws (for a historical review on Gestalt psychology, see Wagemans, 2015a; for extensive reviews of a century of research, see Wagemans et al., 2012a, b). In the experiments below, we will mainly focus on factors of proximity, orientation, and colour similarity (for some relevant psychophysical studies, see Claessens and Wagemans, 2005, 2008; Kubovy and van den Berg, 2008; Kubovy and Wagemans, 1995; Kubovy et al., 1998). In addition, perceptual organisation also encompasses richer, more complex phenomena such as perceptual multistabilities, perceptual hier-archies of local and global levels of organisation, references frames, structural frameworks or skeletons, as well as dynamics and tension, and even perceived causality, animacy, and intentions. It is obvious that we cannot cover a full review of this rich domain of perception here (for extensive reviews, see Wagemans, 2015b, 2018).

Empirical aesthetics or psycho-aesthetics is the branch of science, started by Gustav Fechner (1801-1887), aimed at uncovering the factors that deter-mine aesthetic appreciation, in an attempt to move beyond centuries of philo-sophical speculation (by Plato, Aristotle, Thomas Aquinas, Leibniz, etc.) about the role of proportion, harmony, order, symmetry, integrity, clarity of form, perfection, and unity in variety. In Fechner's hands, the goal was mainly to establish quantitative relationships between (formal) aspects of the stimulus manipulated experimentally and the degree of appreciation (e.g., a point on a rating scale). Nowadays, Fechner's reductionist approach has been expanded in several ways. It now also deals with determinants of aesthetic appreciation of complex objects and events, such as real artworks, in rich contexts such as museums (e.g., Carbon, 2020; Pelowski et al., 2017a). Aesthetic appreciation entails more than beauty, however. It is also about pleasure, interest, wonder, fascination and the sublime, for instance, and it comprises all evaluative aspects, positive or negative, from beautiful to ugly, from pleasure to aversion and disgust (e.g., Fingerhut and Prinz, 2018; Graf and Landwehr, 2017; Marković, 2012; Silvia, 2009). It also goes beyond merely quantitative research aimed at establishing universal laws, by studying proper aesthetic experiences of individuals and explicitly considering individual differences arising from different viewing modes, perceptual styles, personality, familiarity with the stimuli or task, expertise, and knowledge of art. Contemporary research in empirical aesthetics includes a wide range of outcome variables such as pref-erences, rating scales, adjustments, sorting, matching, questionnaires, inter-views, observation, eye tracking, psycho-physiological measures, etc. In a sense, empirical aesthetics is as old as psychology (with Fechner as the found-ing father of both psycho-aesthetics and psychophysics) and equally broad in terms of its variables, manipulations, measurements, and processes of interest. 
Indeed, all contemporary theories of aesthetic experience emphasise the complex interplay between perception, cognition, and emotion (e.g., Pelowski et al., 2017b; Redies, 2015). It is obvious that we cannot cover a full review of this rich domain of science here (for brief introductions, see Brielmann and Pelli, 2018; Jacobsen, 2006; for extensive reviews, see Palmer et al., 2013; Shimamura and Palmer, 2012; Tinio and Smith, 2014).

We will now review the most relevant empirical aesthetics literature on order, harmony, balance, and visual rightness, which is most directly relevant to our current study.

\subsection{Order and Harmony}

Symmetry, an obvious kind of order and regularity, has often been linked to harmony and beauty (for a review, see McManus, 2005). Although symmetric patterns are generally found to be more beautiful (e.g., Jacobsen and Höfel, 2002), recent research shows that the extent to which this is the case depends on the type of task and the level of expertise of the participants (Leder et al., 2019; Weichselbaum et al., 2018). More sophisticated perceivers of abstract patterns or artworks do not always prefer the easiest types of order or regu-larity, such as vertical mirror symmetry, which they often find too rigid and sterile. Instead, they tend to prefer more dynamic and less obvious types of order. This relates to the idea that order and complexity need to be in balance (for a review, see Van Geert and Wagemans, 2020). Serrao (2019) has recently argued that several emergent properties, such as coherence, symmetry, har-mony and consonance, can all be embraced under a more general definition of 'regularity' and he has proposed two explanations, rooted in evolution, of their universal aesthetic appeal. In a recent study with patterns containing all sorts of regularities (created by filling grids with basic tiles that can be rotated; for details, see Westphal-Fitch et al., 2012), Muth et al. (2021) have shown that less obvious order and intentional flaws of perfect order are introduced to make patterns more interesting.

In a series of studies with a particular kind of greyscale abstract computer drawings (created by positioning a large number of originally painted pictorial elements; for details, see Redies et al., 2015), Redies, Hayn-Leichsenring and colleagues have compared the original compositions (intended to be harmoni-ous and ordered), with randomly reshuffled ones in which the same elements occurred but the original compositions were destroyed. In one study, Schwabe et al. (2018) showed that the ratings of harmony and order of these abstract drawings were stable and consistent, even when they were shown for only $50 \mathrm{~ms}$. In a related EEG study, Menzel et al. (2018) obtained a reliable visual mismatch negativity when the original and reshuffled abstract drawings were pitted against one another, even when the observers' attention was drawn away 
from the explicit comparison between the two versions. In combination, these two studies thus showed that the composition in abstract artworks can be detected quickly and automatically by the human brain. In a more recent study with similar abstract compositions, Letsch and Hayn-Leichsenring (2020) showed that artists created images that were less self-similar and closer to the so-called rule of thirds (a principle which is often proposed to make a good composition) than those created by laypersons, and that naïve observers were able to distinguish these two sets better than chance.

\subsection{Balance}

Moving beyond perfect mirror symmetry and other types of rigid order, theories of aesthetics have often emphasised the role of balance. For instance, Arnheim $(1971,1982,2004)$ has argued that a visual artwork is in balance when it has a good organisation and when all attractive forces cancel each other out and everything seems at rest. In many studies of empirical aesthetics, this notion has been interpreted physically, in terms of centre of gravity or centre of mass, with luminance or colour weights as inputs (e.g., Gershoni and Hochstein, 2011; Locher et al., 1998, 2001a; McManus et al., 1985, 2011; on the notion of compositorial 'weight', see Koenderink et al., 2017). For exam-ple, Locher et al. (1998) asked 13 first-year design students to create compo-sitions with a fixed set of elements (circles, squares, rectangles, or leaves) of variable size. They found that the centre of a design almost coincided with the geometric centre of the picture frame, demonstrating the power of the centre to function as a balancing point to anchor the structural skeleton of the com-position. The same study, however, has also considered the more complex and interesting notion of dynamic balance, in which elements are arranged asym-metrically but in such a way that their visual forces compensate one another. Specifically, it was found that the perceived balance ratings correlated nicely with the computed index of dynamic balance. A follow-up study with 32 art-ists (Locher et al., 2001a) replicated and extended these findings with a wider range of basic elements.

More recently, Hübner and Fillinger (2016) compared different objective measures of perceptual balance (e.g., centre of mass, mirror symmetry, and homogeneity) and their relation to aesthetic preference. In general, they found that centre of mass is an adequate measure of perceptual balance but that homogeneity should be included to be able to predict preference. In a follow-up study, Hübner and Fillinger (2019) compared perceptual balance ratings for a wider range of stimuli, such as single-element, multiple-element, and dynamic patterns. While perceptual balance was captured well by the simple physical balance measure in single-element patterns, the perceptual balance of multiple-element and dynamic patterns required a more complex measure of 
gravitational stability. In the most recent study of this series, Fillinger and Hübner (2020) investigated the role of perceptual balance and perceptual stability in Japanese calligraphies. Replicating the earlier findings of Gershoni and Hochstein (2011) with a different method, they showed that perceptual balance measures were uncorrelated with liking ratings. In a second experiment, stability and one of the balance measures did correlate with liking, but only for atypical calligraphies. This line of work demonstrates that the role of balance can vary with the type of pattern (from simple to complex, perhaps even interacting with familiarity).

\subsubsection{Balance in Mondrians}

Several empirical studies have been conducted with digital copies and manipulations of abstract paintings made by Piet Mondrian during his period of Neoplasticism, for which he is best known. As part of the De Stijl art movement, this nonrepresentational art form was intended as the new 'pure' plastic art. In order to create universal beauty, Mondrian believed it was necessary to limit the formal vocabulary of painting to three primary colours (red, yellow, and blue), three primary lightness values (black, white, and grey) and two primary orientations (horizontal and vertical). Mondrian himself experimented extensively to create balanced compositions with these primitives during the 1920s and 1930s, studying and revising them in his studio, often moving lines around by small distances.

McManus et al. (1993) asked art and non-art students to indicate their preference between digital copies of 25 original Mondrians and two versions derived from the originals by displacing the black lines slightly. Overall, the original Mondrians were preferred to a greater extent than predicted by chance. Somewhat surprisingly, non-art students discriminated original from per-turbed Mondrians slightly more accurately than art students, although there was a good deal of variability between participants and stimuli. In a related study, Latto et al. (2000) showed that non-art students preferred Mondrians in the original orientation over those in eight different oblique orientations. Both of these studies provided empirical support for Mondrian's choice of exactly those compositions in the final version of the paintings (i.e., the notion of 'visual rightness'; see below).

Locher et al. (2005) have extended this work by investigating the contribu-tion of colour and size of the three areas painted red, yellow, and blue to the perceived weight of these areas and the perceived location of the balance cen-tres in six original Mondrians and five variations. Specifically, design-trained and untrained participants rated the apparent weight of each coloured area and determined the location of the balance centre of each composition. For both groups of participants, the perceived weight of the red and yellow areas varied as a function of their size, and the perceived location of the balance centre was 
judged more consistently in the original Mondrians as compared to the manipulated versions. Only the design-trained participants, however, perceived significant shifts in balance centres from the original to the manipulated versions. In sum, this study demonstrates that colour and area affect the weight relationships in abstract compositions, and their role in perceived balance, at least for participants with some design training.

\subsubsection{Balance and Visual Rightness}

A related concept to that of balance is that of visual rightness, which refers to the idea that there is a right way to arrange the elements into a compo-sition with an optimal effect on the viewer, and the idea that artists know how to achieve this (cf. Mondrian). In an interesting series of studies on this topic, Locher and his colleagues have provided empirical support for these two ideas. First, in relation to the earlier work on balance, Locher and Nagy (1996) have used different versions of the black-and-white plates from the Maitland Graves Design Judgment Test and from the Meier Art Tests, which were bal-anced to different extents, as well as colour reproductions of eight balanced abstract and representational paintings by famous artists such as Kandinsky, Sargent, Schwitters and Vuillard, and different versions which were made less balanced by displacing one or two pictorial elements to a new location which was not part of the structural framework. They presented these to art-trained and untrained participants for $100 \mathrm{~ms}$ or $5 \mathrm{~s}$, asking them to rate the composi-tions for balance on a six-point Likert scale. Results showed that both groups of participants were able to discriminate between the less balanced and the more balanced versions of the black-and-white plates within $100 \mathrm{~ms}$. They could also discern differences in balance between the different colour paint-ings within $100 \mathrm{~ms}$, but not between the original and modified versions of each painting. These findings support the view held by artists and art theorists like Arnheim that a visually right composition has an efficient structural organisa-tion that is salient to viewers and can be captured in a single glance.

Locher et al. (1996) have provided further empirical support for this view by showing that disruption of the balanced organization of abstract and representational paintings led to reliably shifted locations of the perceived balance centres of the originals compared to the experimentally perturbed, less bal-anced versions, both for design and museum professionals as well as for indi-viduals untrained in the visual arts. With the same stimuli, Locher et al. (1999a) showed that design professionals were significantly better at detecting the original versions than participants untrained in the visual arts (hit rates of 64\% and 55\%, resp.). In another experiment, participants were asked to posi-tion a major structural element that was removed from each of the six paint-ings at the location where they thought it appeared in the original. Trained and 
untrained participants were in good agreement about the general location, reflecting the overall compositional structure, but not precisely its actual location. In a third study, Locher (2003) used four abstract and four representa-tional paintings by renowned artists such as Hofmann, Avery, Valloton and Serusier, and two versions in which one or more elements were displaced in ways that preserved or destroyed the primary structural organisation of the composition. It was found that naïve participants (psychology students) could discriminate between the originals and the strongly perturbed, but not the slightly altered version, than expected by chance. In addition, for abstract works, the hit rates were highest when their compositional style and form were the focus of attention, while for representational works performance was best when participants focused on their content and realism. In general, this line of work shows that a visually right composition is salient to viewers, even with-out formal training in the visual arts, although there is a range of tolerance around what is visually right and more subtle deviations cannot always be noticed, especially not by participants who are not trained in the arts.

\subsection{Other Relevant Topics}

Following this review of the most relevant literature on order, harmony, bal-ance, and visual rightness, we now want to briefly point to a few other topics which are also somewhat related to the issues addressed here. There is an extensive literature on the detection of symmetry as well as some deviations of symmetry, such as skewed symmetry (e.g., Wagemans, 1993, 1997; Wagemans et al., 1993), often studied in the context of shape deformations and invari-ances under transformations (e.g., Van Gool et al., 1994; Wagemans et al., 1994, 1997). However, this topic has not been studied in relation to aesthet-ics, as far as we know. More recently, Wright and Bertamini (2015) did study aesthetic preference for dynamic symmetry, comparing also different global transformations such as translation, rotation, expansion, and shear. Regarding shape, Pinna has conducted interesting phenomenological and empirical work on the higher-order relationships between shape and meaning (Pinna, 2010, 2011) and between shape and colour (Pinna and Deiana, 2015).

There is an extensive literature on special relational factors in composition, such as the golden section and the rule of thirds. The current consensus seems to be that, despite their proclaimed importance in art and photography theory, both of these principles play only a minor role, if any, when evaluated thor-oughly in empirical studies with preference and rating measures (e.g., Amirshahi et al., 2014; Boselie, 1992), or that they are, at least, very sensitive to the specific research methods (e.g., Green, 1995) and subject to substantial individual differences between observers (e.g., McManus and Weatherby, 
1997). On the related topic of reference frames and how to position significant elements on special points or axes, there is also an extensive literature. Here, it seems that one finds significant effects of a special internal structure when studied with simple geometric compositions (e.g., Guidi and Palmer, 2015) but that they are much less obvious in real artworks. For instance, in a recent study with series of 19th-century Japanese prints (e.g., Hokusai's Thirty-six views of Mount Fuji), Bruno (2020) found very little support for framing effects according to power lines or points defined by well-known composition principles. Instead, he found evidence for an overall bias for moderate asym-metry, although this was also subject to individual and cultural differences.

Regarding the structural skeleton of shapes, the notion of a medial axis has become quite important in computer vision and psychophysics. In an extensive review paper, with lots of visual examples, Fol Leymarie and Aparajeya (2017) have demonstrated the practical use of these techniques to study the production, perception and appreciation of shapes in artworks as diverse as drawings, paintings (e.g., Picasso, Matisse), calligraphy, and graffiti, including dynamic aspects such as tension. In a related paper, Zhu (2016) has developed a theory of visual tension created by distortions of basic shapes, inspired by the physics of elastic tension, and has applied this to the perception and appreciation of Eye in eye, a painting by Edvard Munch, and Joachim's Dream, a fresco by Giotto.

\subsection{Summary of Five Key Findings and Implications for Our Research}

The key findings from this broad and highly varied set of studies have some implications for our own study on Malevich's Black Square and Red Square. These can be summarised as follows:

1. Observers can detect small perturbations from perfect regularity (e.g., per-fect mirror symmetry) and shape deviations or transformations. We will test whether participants can discriminate between the original, slightly perturbed square and a perfect square.

2. Observers tend to prefer imperfect order (e.g., moderate asymmetries) and more complex, dynamic types of regularity over completely perfect order and simpler, more static types of regularity. We will test whether participants prefer Malevich's constellation with a perturbed square over a perfect square.

3. Artists often strive for compositions which are balanced and harmonious. Artists have strong intuitions (and may have learned rules of composition) about how to create balanced and harmonious compositions. Heavier 'weights' due to size or colour of some elements can be compensated by 'weights' of other elements in another part of the display. Dynamic 
balance (e.g., by variable size, colour and orientation of the elements) is more interesting than static balance. We will compare different versions of Malevich's basic configuration and test whether the specific configuration chosen succeeds in compensating best for the slight distortion of the black square.

4. Artists have strong intuitions (and may have learned rules of composition) about which of all possible compositions with the same elements is best (cf. perceptual goodness) or exactly right (cf. visual rightness). We will test whether participants will find Malevich's original composition the most balanced, harmonious, pleasing and interesting amongst all of its variations.

5. In several of the reviewed studies, more artistically sophisticated participants (e.g., design students or art-trained participants) are better at detecting slight perturbations of original compositions than naïve participants. However, other studies show that a good composition can be detected quickly and automatically and that paintings with visual rightness can be discriminated from paintings with small perturbations of their structural organisation at better than chance levels even by participants with no formal art training. We will include art background as a variable of interest in our three experiments.

\section{Experiment 1}

\subsection{Introduction}

In this experiment, the aim was to investigate the effect of the presence and obliquity of the red square on the perception of the obliquity of the black square in Malevich's Black Square and Red Square abstract painting (Fig. 1, left). In this artwork, the black square is put in the standard vertical-horizontal orientation, while the smaller red square is located below and to the right of the black square and is tilted counterclockwise in such a way that its horizontal axis creates an angle of $11^{\circ}$ with respect to that of the black square. The right vertical edge of the black square has a distortion. The red square has a similar but slighter distortion. If we take $\mathrm{V}$ and $\mathrm{H}$ as the absolute vertical and horizon-tal axes of the reference system, respectively, the two horizontal edges of the black square would be parallel to $\mathrm{H}$, while its left vertical edge would be paral-lel to $\mathrm{V}$ but its right vertical edge is tilted from $V$ by $2^{\circ}$ such that $\alpha=90^{\circ}, \beta=92^{\circ}$, and $\beta^{\prime}=88^{\circ}$. If we take $\mathrm{V}^{\prime}$ and $\mathrm{H}^{\prime}$ as the relative vertical and horizontal axes aligned to the small red square, respectively, the red square would have two horizontal edges parallel to $\mathrm{H}^{\prime}$, while the vertical edges are roughly paral-lel to $\mathrm{V}^{\prime}$ (the right edge too has a slight inclination). The right edge of the red 
square, if extended, would meet the top right-hand corner of the black square (Milner, 1996). Instead of focusing on the symmetries and golden sections discussed by Milner (1996), we wanted to investigate how the main stimulus and participant factors influenced the detection of the geometrical deviation from the exact square (Fig. 1, right). Specifically, we created versions with per-fect and distorted black and red squares in different combinations and asked observers with variable degrees of training in art to determine whether the black square was a perfect geometrical shape or not. We hypothesised that the presence of the red square in Malevich's original artwork served to reduce the perceived distortion in the black square.

\subsection{Methods}

\subsubsection{Participants}

Two groups of students participated in this experiment: 32 graduate art students from Tarbiat Modares University (Age: mean $=27.2$ years, $\mathrm{SD}=6.8$ years, range $=21-36$ years; Gender (Note 3): 17 females, 15 males) and 23 high-school students (Age: mean $=15$ years, $\mathrm{SD}=0.50$ years, range $=14-16$ years; Gender: 11 females, 12 males). In Group 1, all participants were educated in visual arts, and could be considered an art expert group, but note that they are also older on average. In contrast, in Group 2, none of the participants had any educational nor practical experience in arts, and could be consid-ered an art novice group, although they were considerably younger on aver-age. Participants were asked to fill out a questionnaire, including information about gender, age, handedness, education, and field of study. All participants had normal or corrected-to-normal vision. The experiment was performed in the Persian language. All core variables and concepts used in this study are provided in both Persian and English in Supplementary Table S1. This experiment was approved by the Social and Societal Ethical Committee of Tarbiat Modares University, Tehran, Iran, and all participants (and their parents if they were younger than 18) gave explicit informed consent prior to their participa-tion in this experiment.

\subsubsection{Stimuli}

We used a digital version of the original artwork Black and Red Square by Malevich (1915) plus five alternatives obtained by modifying the reference work (see Table 2 and Fig. 4). In the six stimuli, the black square was either the original version or it was replaced with an exact geometric version of the same size. Although the red square has only a very slight distortion, it too was replaced with an exact geometric version of the same size. Thus, in six stimuli, the black square was either original or exact, and the red square was either original, or exact, or absent. 
Table 2.

Number of participants who perceived the black square as an exact square for each of the six stimuli in Experiment 1.

\begin{tabular}{llllcr}
\hline & & & \multicolumn{3}{c}{ \#Yes } \\
\cline { 4 - 6 } Stimulus & Black square & Red square & Overall & Group 1 & Group 2 \\
& & & $\boldsymbol{N = 5 5}$ & $\boldsymbol{N}=\mathbf{3 2}$ & $\boldsymbol{N}=\mathbf{2 3}$ \\
& & & $\boldsymbol{n ( \% )}$ & $\boldsymbol{n}(\boldsymbol{\%})$ & $\boldsymbol{n ( \% )}$ \\
\hline Stimulus 1 (Fig. 4a) & Original & Original & $45(81.8)$ & $27(84.4)$ & $18(78.3)$ \\
Stimulus 2 (Fig. 4b) & Original & Exact & $16(29.1)$ & $12(37.5)$ & $4(17.4)$ \\
Stimulus 3 (Fig. 4c) & Exact & Original & $43(78.2)$ & $24(7.5)$ & $19(82.6)$ \\
Stimulus 4 (Fig. 4d) & Exact & Exact & $46(83.6)$ & $25(78.1)$ & $21(91.3)$ \\
Stimulus 5 (Fig. 4e) & Original & Absent & $18(32.7)$ & $10(31.2)$ & $8(34.7)$ \\
Stimulus 6 (Fig. 4f) & Exact & Absent & $52(94.5)$ & $30(93.8)$ & $22(95.6)$ \\
\hline
\end{tabular}

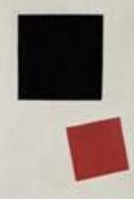

(a)
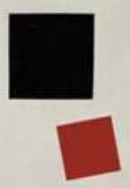

(b)

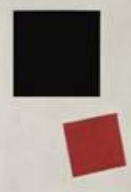

(c)
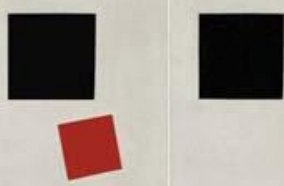

(d)

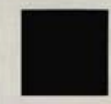

(f)

Figure 4. Experimental variations of Black Square and Red Square used in Experiment 1. (a) Original artwork; (b) original black square and exact-geometric red square; (c) exact-geometric black square and original red square; (d) exact-geometric black and red squares; (e) original black square only; (f) exact-geometric black square only.

\subsubsection{Apparatus}

Experiment 1 was carried out in a testing room at Tarbiat Modares University, Tehran, Iran. All sessions took place in the same room, maintaining the same conditions. The testing room was quite dark with only a small amount of natural light. All the stimuli were displayed on a 19-inch monitor screen (Generic PnP, ASUS, Suzhou, China, resolution: $1366 \times 768$ pixels, refresh rate: 85 $\mathrm{Hz}$ ). Participants were seated $70-100 \mathrm{~cm}$ from the monitor with upright head. The height of the seat was adjusted to align the screen centre to each participant's eyes. The stimuli were presented at a size of $175 \mathrm{~mm} \times 98 \mathrm{~mm}$ (approximate visual angle of $14.25^{\circ} \times 8.00^{\circ}$ ).

\subsubsection{Task and Procedure}

For each stimulus, the observer was asked to record how they perceived the black square in the stimuli. Specifically, they had to answer the following question: is the black square a geometrically exact square or does it have some 
obliquity? They could respond by one of two choices: Yes (= exact square) or No (= oblique square). After the instructions, the experiment started, and the fixation cross became visible for $500 \mathrm{~ms}$ immediately preceding the first image, which was displayed for a fixed duration of $2000 \mathrm{~ms}$. Immediately afterwards, each stimulus was followed by a two-choice Yes/No question. Then, this sequence continued with the next stimulus and so on to the last one. All stimuli were presented in fully randomised order. The experiment lasted around 10 minutes. This included the time spent to instruct the participants but not the time to complete the questionnaire.

\subsection{Results}

Table 2 reports the number of participants who perceived the black square as an exact square for each of the six stimuli. The baseline conditions, with-out the red square, show that almost all participants (52/55) did perceive the exact square (Stimulus 6) correctly, while only a minority of the participants (18/55) perceived the original black square (Stimulus 5) mistakenly as exact (McNemar's $X^{2}=30.42, p<0.001$; effect size: $\varphi=0.53$ ). A clear context effect was observed due to the presence of the small red square when compar-ing the number of incorrect responses to Stimulus 1 (the original work) with those to Stimulus 5 (45 18, McNemar's $X^{2}=25.1, p<0.001$; effect size: $\varphi=0.48$ ), reflecting the fact that the distortion is masked by the red square, as hypothesized. Also, the context effect disappeared when we replaced the red square in the original work with an exact red square (45 16, McNemar's $X^{2}=27.1, p<0.001$; effect size: $\varphi=0.50$ ), clearly indicating that the masking effect is due to the very subtle distortion in the red square. However, a comparison between Stimuli 3 and 4 showed almost no context effect with respect to the obliquity of the red square when the black square was replaced with an exact black square (43 46, McNemar's $X^{2}=0.44, p=0.50$; effect size: $\varphi=0.06$ ).

We observed no statistically significant differences between Group 1 (with more experience in arts) and Group 2 when we compared, for each stimulus, how often they perceived the black square as an exact square. To investigate if the group with greater art experience (Group 1) was better at this task than the art novice group (Group 2), we counted the total number of correct answers (i.e., instances where the exact black square was perceived as an exact square and instances where the oblique black square was perceived as an oblique square). Contrary to what we expected, the older, more experienced partici-pants in Group 1 did not outperform the younger, less experienced participants in Group 2: Group 1 participants provided correct answers in 126 out of 192 instances (32 participants $\times 6$ stimuli), while Group 2 participants responded 
correctly in 101 out of 136 instances (23 participants $\times 6$ stimuli), proportions $(0.660 .74)$ that do not differ statistically $\left(X^{2}=2.78, p=0.09\right.$; effect size: $\varphi=0.09)$.

\subsection{Discussion}

The results of this first experiment are very clear: they confirm the effects of the stimulus factors, in the absence of an expertise effect. Specifically, the presence of the small, distorted red square reduces the noticeability of the obliquity of the large black square. Even though the distortion in the red square is subtle, the perfect square does not yield the same effect. The two groups of participants, graduate art students and high-school students, did not differ in how well they could discriminate the exact from the oblique black squares. It is possible that the groups did not differ enough in terms of art expertise, or that reduced expertise in art in the novice group was compensated by slightly better acuity which usually accompanies younger age. (Note that all participants had normal or corrected-to-normal vision but we did not measure their acuity.)

\section{Experiment 2}

\subsection{Introduction}

In Experiment 1, the simultaneous presence and obliquity of the red square masked the distortion of the original black square. The aim of Experiment 2 was to investigate in more detail how the different geometric components of the configuration formed by the black and red square affected the detectability of the obliquity of the black square in Malevich's Black Square and Red Square. Specifically, we modified a digital copy of the original artwork to create three sets of stimuli manipulating the degree of skewness of the black square's side edge, the proximity of the red square to the black square, and the orientation of the red square, respectively. In addition, we also tested the role of colour similarity by repeating the same manipulations with a small black square replacing the small red square.

In Experiment 1, there was no difference between participants with more experience (Group 1) and participants with less experience (Group 2) in their perception of the distortion of the black square. Perhaps the groups were not large enough, or we did not have sufficient control over the nature of their art expertise, or expertise was confounded by age. Thus, in Experiment 2, we had a larger group of participants of the same age and separated them based on some known determinants of art expertise. While art education has been used as a measure of art expertise in the majority of studies in empirical aesthetics 
and art perception, it is important to note that art expertise might not only be defined as being educated in arts and the ability of making or creating an artwork (e.g., Chamberlain and Wagemans, 2015; Leder et al., 2014; Rosenberg and Klein, 2015; Winston and Cupchik, 1992). Indeed, several methods have been used in the literature to define and quantify art expertise (e.g., Bimler $e t$ al., 2019; Silvia, 2007; Specker et al., 2020). To measure the art expertise of our participants, we therefore created a questionnaire consisting of three parts (see Section 3.2.2. Questionnaire).

\subsection{Methods}

\subsubsection{Participants}

Ninety-five participants were involved in this experiment (Age: mean $=22.65$ years, $\mathrm{SD}=7.65$ years, range $=13-39$ years; Gender: 44 females, 51 males). All participants had normal or corrected-to-normal vision. Participants were asked to fill out a generic questionnaire including information about gen-der, age, handedness, education, field of study, as well as a custom-made art expertise questionnaire tapping into art experience, knowledge, and interest. The experiment was performed in the Persian language. All core variables and concepts used in this study are provided in both Persian and English in Supplementary Table S1. This study was approved by the Social and Societal Ethical Committee of Tarbiat Modares University, Tehran, Iran, and all participants (and their parents if they were younger than 18) gave explicit informed consent prior to their participation in the experiments.

\subsubsection{Questionnaire}

The questionnaire to assess art expertise consisted of three parts. Part A was designed to measure art experience using a mix of seven common items derived from former studies (Chamberlain and Wagemans, 2015; Leder et al., 2014; Locher et al., 1999b, 2001b; Winston and Cupchik, 1992). Part B was designed to measure art knowledge. This part consisted of 10 items originally proposed by Smith and Smith (2006) to assess art fluency. As we are mainly focused on abstract art in this study, we added five items in which participants were asked to identify the style and artists of abstract artworks. Part $\mathrm{C}$ was designed to measure art interest. This part consisted of 11 items derived from the VAIAK questionnaire (Specker et al., 2020). All items were rated on a five-point scale. The questionnaire is made available in the Supplemental Material, together with an index of its reliability, which was good (see Supplementary Appendix S1). For each participant, art experience, art knowledge, and art interest scores were calculated by summing up the ratings given to the indi-vidual questions within the associated part of the questionnaire. Participants 
were assumed to be experienced, knowledgeable, or interested in art, if their associated scores were greater than or equal to half of the maximum possible score in each part.

\subsubsection{Stimuli}

In total, 124 stimuli were created, divided over six subsets, three with a small red square (R) and three with a small black square (B; see Fig. 5).

Set $1 R$ was focused on the effect of Skewness of the black square's side edge (14 stimuli). We skewed the right edge (the originally oblique edge) of the black square in 13 steps of $0.5^{\circ}$ increments in positive and negative directions (see Fig. 5a). Hence, the obliquity of the right edge was $-2.0^{\circ},-1.5^{\circ}$, $-1.0^{\circ},-0.5^{\circ}, 0.0^{\circ}, 0.5^{\circ}, 1.0^{\circ}, 1.5^{\circ}, \mathbf{2 . 0}^{\circ}, 2.5^{\circ}, 3.0^{\circ}, 3.5^{\circ}, 4.0^{\circ}, 4.5^{\circ}$, with respect to the vertical axis; note that the obliquity in the original painting was $2.0^{\circ}$ (marked in boldface).

Set $2 R$ was focused on the effect of Proximity (34 stimuli). It consists of four subsets (see Fig. 5b): subset 1 with seven distance changes along the absolute vertical axis (V), subset 2 with ten distance changes along the absolute hori-zontal axis $(\mathrm{H})$, subset 3 with seven distance changes along the relative verti-cal axis $\left(\mathrm{V}^{\prime}\right)$, and subset 4 with ten distance changes along relative horizontal axis $\left(\mathrm{H}^{\prime}\right)$. In each subset, the translations were such that the intercentroid arith-metic distance of the two squares along the associated translation axis was equal to its original value multiplied by each of the translation factors reported in Table 3.

Set $3 R$ was focused on the effect of Orientation of the red square (14 stimuli). It included rotations of the red square, with the bottom edge at angles of $0^{\circ}, 5.5^{\circ}, 1^{\circ}, 16.5^{\circ}, 22.5^{\circ}, 30^{\circ}, 37.5^{\circ}$ and $45^{\circ}$ clockwise (CW) and counterclockwise (CCW) with respect to the $\mathrm{H}$ axis (see Fig. $5 \mathrm{c}$ ).

Sets $1 B, 2 B$, and $3 B$ were focused on the effect of the small square's Colour (62 stimuli). The stimuli were the same as those used in Sets 1R-3R. However, to study the effect of colour similarity, we changed the colour of the small square from red to black (see Fig. 5d).

\subsubsection{Apparatus, Task and Procedure}

These were all the same as in Experiment 1 except for the following points. All stimuli were generated in Matlab (MathWorks, Natick, MA, USA) and an interface was developed using Matlab GUI 2018 to display the stimuli. All stimuli were presented in fully randomised order. The experiment lasted between 20 and 30 minutes (including voluntary breaks). This included the time spent to instruct the participants but not the time to complete the questionnaire. 


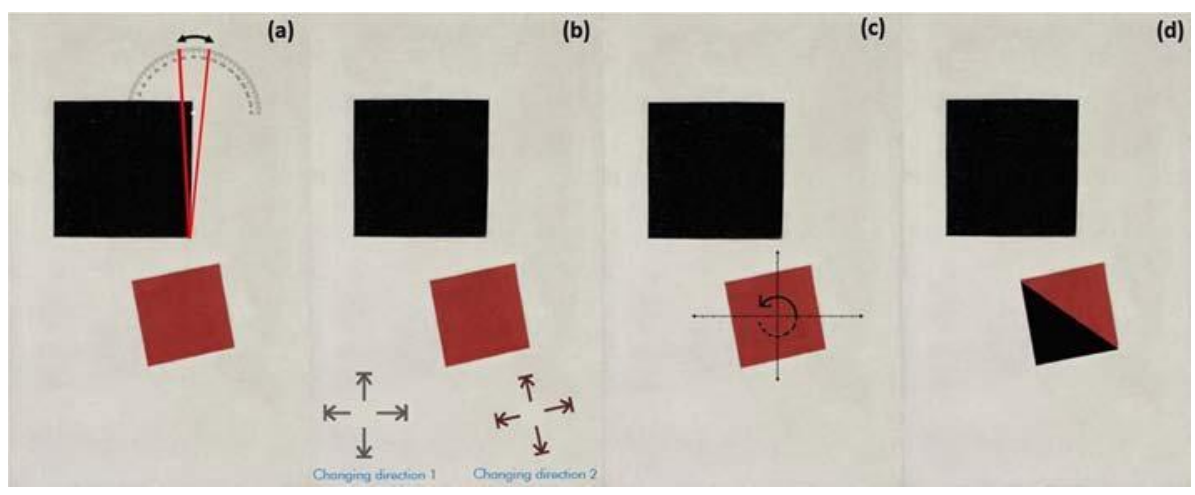

Figure 5. Four schematic displays illustrating the different factors manipulated in the six sets of stimuli in Experiment 2. (a) Skewness of the big black square's edge; (b) proximity of the small red square to the big black square (by translating it in absolute or relative direction); (c) orientation of the small red square; and (d) colour similarity (small square = red or black in half of the stimuli).

\section{Table 3.}

The axis of translation and the translation factors for the four subsets of stimuli in Set 2 .

The original values are marked in boldface.

\begin{tabular}{lll}
\hline Subset & $\begin{array}{c}\text { Axis of } \\
\text { translation }\end{array}$ & Translation factors \\
\hline
\end{tabular}

1

Absolute vertical translation

$0.85, \mathbf{1 . 0 0}, 1.15,1.30,1.45,1.6,1.75$

2

Absolute horizontal translation

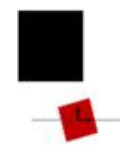

$-0.80,-0.50,-0.20,0.10,0.40$,

$0.70,1.00,1.30,1.60,1.90$

3

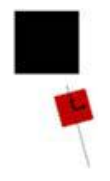

$0.85, \mathbf{1 . 0 0}, 1.15,1.30,1.45,1.6,1.75$

Relative vertical translation

4

Relative horizontal translation

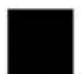
$-2.60,-2.00,-1.40,-0.80,-0.20$, $0.40,1.00,1.60,2.20,2.80$



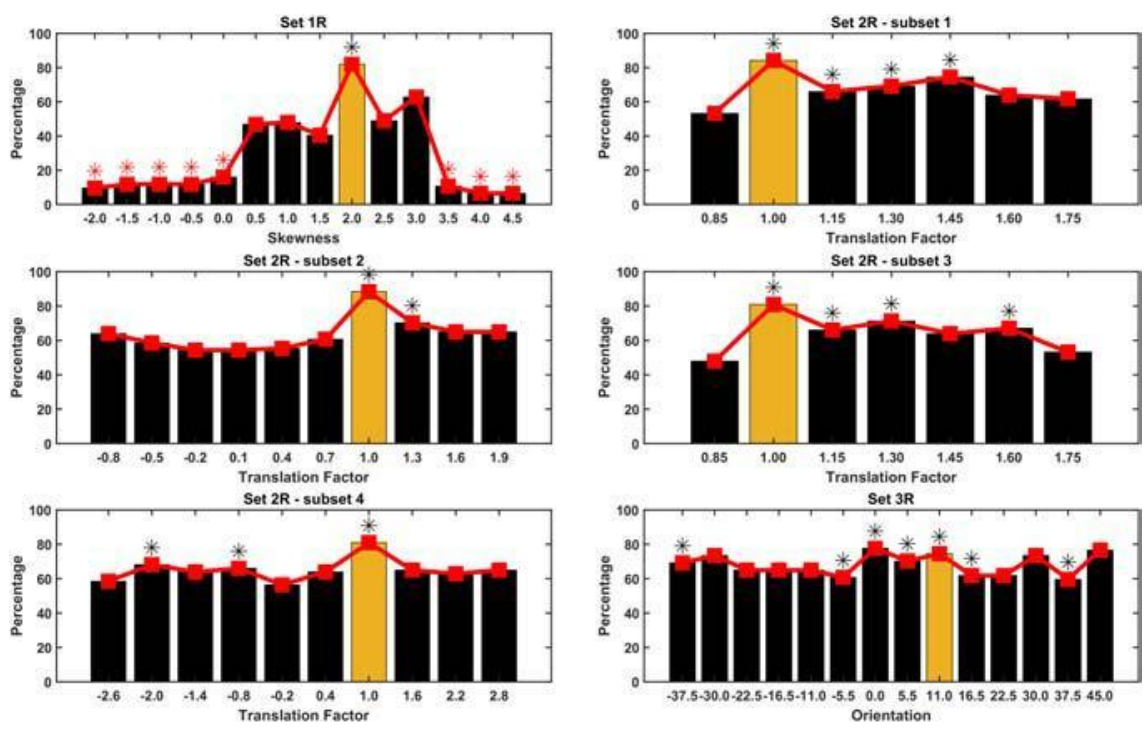

Figure 6. Visual perception of the black square in the presence of a small red square (subsets $\mathrm{R})$. Each bar shows the percentage of the participants who perceived the large black square as an exact-geometrical one in each stimulus. The yellow bars indicate the original version of Malevich's work in each set/subset. Black/red stars mark percentage values significantly greater/smaller than chance level (50\%), respectively, based on the chi-squared two-proportions test.

\subsection{Results}

Figure 6 shows the effects of skewness of the black square, proximity and orientation of the red square on the perception of the black square as an exact square. Overall, the skewness of the black square and the proximity of the two squares showed a substantial effect on the perception of the black square, while the orientation of the red square did not seem to affect the perception of the black square all that much; the percentages of Yes responses hovered between $60 \%$ and $80 \%$ for the full range. Examining the effects of skewness and prox-imity, the likelihood of the black square being perceived as an exact square was always maximum in the original configuration. Pairwise chi-squared two-proportions tests between the percentage of Yes responses for the original configuration and that for all the alterations showed significant differences $(p<0.05)$ in Set $1 \mathrm{R}$ and Set 2R (subsets 1-4; see Supplementary Fig. S1). As shown in Fig. 6 (top left panel), the effect of the obliquity of the black square was tuned narrowly around the original value, with a significant drop to chance levels for a relatively broad range of values between $0.5^{\circ}$ and $3.0^{\circ}$ and then a further drop to correctly picking up the distortion outside this range. Regarding proximity (four panels labelled $2 \mathrm{R}$ ), we found that the likelihood 
of the black square being perceived as an exact square dropped significantly when the red square was located closer to the black square (left of the yellow bar) or further away (right of the yellow bar) compared to the original artwork, with a slightly larger drop when closer, especially for subsets 1 and 3 (vertical translations).

In sets $1 \mathrm{~B}-3 \mathrm{~B}$, when the small red square was replaced with a black square of the same size, the likelihood of detecting the obliquity of the large black square increased overall, i.e., the number of participants perceiving the black square as an exact square dropped. This effect was particularly significant when we compared the original artwork (Fig. 6, yellow bars) with stimuli obtained by only replacing the small red square in the original work with a black one (Fig. 7, yellow bars).

To study the effect of art experience on the perception, we performed subgroup analyses on participants with different levels of art experience, art knowledge, and art interest. Although the proportions of participants who failed to perceive the obliquity of the black square were slightly higher with less art knowledge, no statistically significant effect of art experience on the perception of the black square was observed in any of the different subsets of sets $1 \mathrm{R}-3 \mathrm{R}$ and sets $1 \mathrm{~B}-3 \mathrm{~B}$.
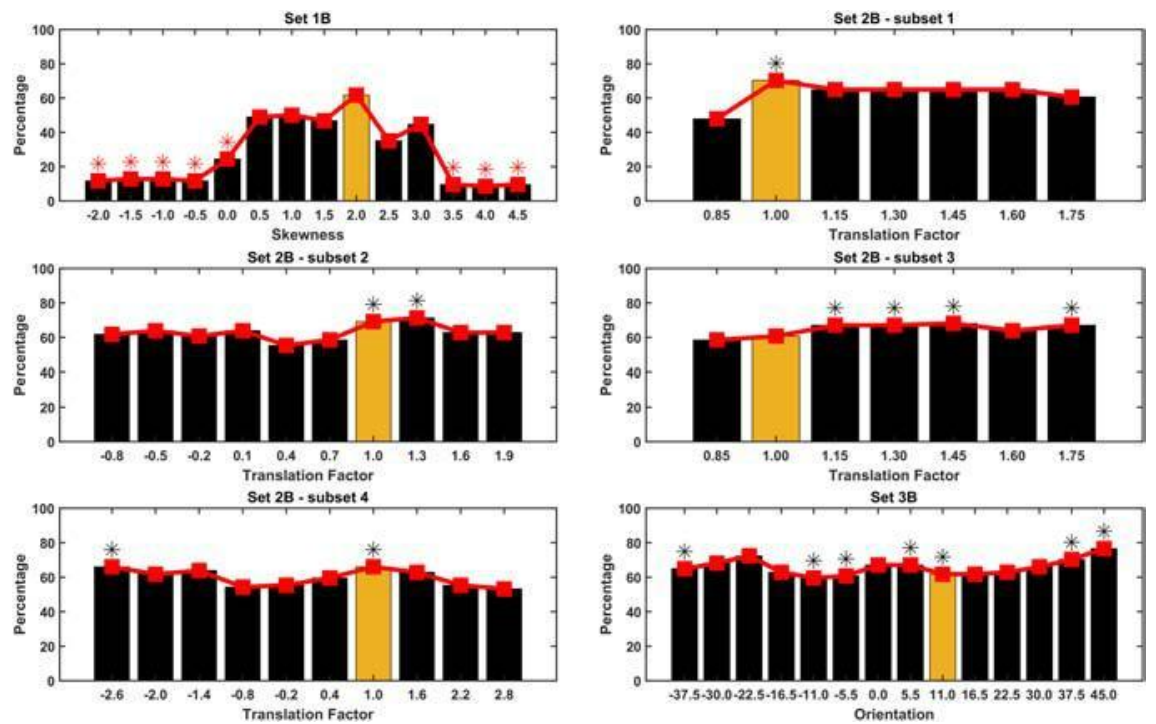

Figure 7. Visual perception of the black square in the presence of a small black square (subsets B). Each bar shows the percentage of the participants who perceived the large black square as an exactgeometrical one in each stimulus. The yellow bars indicate the original version of Malevich' work in each set/subset, albeit with the small red square replaced with a black square of the same size. Black/red stars mark percentage values significantly greater/smaller than chance level $(50 \%)$, respectively, based on the chi-squared two-proportions test. 


\subsection{Discussion}

Participants were generally not doing well in this task. Because only the $0^{\circ}$ skew stimulus in sets $1 \mathrm{R}$ and $1 \mathrm{~B}$ was geometrically an exact square, all per-centages above 50\% indicate that participants were mistakenly perceiving the distorted black square as an exact one, while percentages below $50 \%$ (except for the $0^{\circ}$ skew) indicate that participants were indeed detecting the distor-tion. Overall, we found that the distortion was most often missed in the origi-nal configuration, providing strong support to the art-historical intuition that Malevich chose this particular configuration to mask the obliquity of the large black square. This was especially the case with the original degree of obliquity (skewness) and the original distance between the large black square and the small red square. This masking effect was tuned rather narrowly around the original skew. With obliquity angles below $0.5^{\circ}$ and above $3.0^{\circ}$, the distortion became noticeable; even the perfect square was seen as imperfect. The mask-ing effect also depended clearly on the separation between the large black square and the small red square. At the original distance, the distortion was missed in the majority of cases, while shorter and longer distances gener-ated significantly fewer incorrect Yes responses (although still higher than chance level in many of the conditions). Bringing the small red square closer to the large black square in the vertical direction caused a larger disruption of the masking effect than increasing the vertical separation. The horizontal translations generated a relatively robust masking effect with little or no dif-ference between translations putting the small square more below the large black square (leftwards translations) or further to the right of it (rightward translations). The orientation of the red square did not seem to affect the per-ception of the black square as exact or distorted all that much. So, Milner's (1996) intuition about the possible relevance of the exact compositional geom-etry, namely that the right edge of the red square, if extended, would meet the upper right-hand corner of the black square, does not receive much empirical support. When the small red square was replaced by a small black square, the masking effects were significantly reduced, confirming the role of colour con-trast as an important factor. In other words, when the large and small square were both black, it became easier to detect the obliquity when it was present, while closer to chance level responses were given in all other cases.

In summary, Malevich's choice of positioning a small red square at a particular distance from the large black square, and in a particular orientation, was very instrumental in compensating for its obliquity. The systematic manipulations of Experiment 2 revealed that skewness itself was the strongest factor driving the perception of the black square as exact or distorted. Proximity was also important, especially in the vertical direction. The horizontal separa-tion between the two squares and the orientation of the red square were less 
important. The moderating role of these geometric relations was strongest when the small square was red. To what extent these factors also affected the aesthetic evaluation of the configuration as a whole rather than the perception of the large black square's shape as such, was examined in the next experiment.

\section{Experiment 3}

\subsection{Introduction}

In both experiments so far, we have investigated whether the large square in Malevich's Black Square and Red Square, which is actually distorted, is correctly perceived as distorted or misperceived as being a geometrically exact square. Overall, we found that the distortion was most often missed in the original configuration, confirming the idea that Malevich wanted to induce a purely subjective (i.e., non-objective) perception of a perfect square by means of a 'good' composition, with the proper balancing forces generated by the spatial and colour relations between the large black square and the small red square, in order to compensate for the intentional (objective) imperfec-tions. The perceptual effects are clear. In the present experiment, we want to go one step further by also investigating the aesthetic evaluation of the original composition relative to its experimental variants. This additional step seems essential to empirically validate whether the two central principles of Suprematism - (1) the focus on nonobjective entities and dynamic forces and (2) the supremacy of the feelings generated by minimal compositions - somehow resonate with what perceivers of a Suprematist artwork report about their aesthetic appraisals and feelings. In Experiment 3, we therefore asked participants to indicate how pleasing, interesting, balanced and harmonious they find the original reference work by Malevich and the modified versions of sets 1R-3R and 1B-3B used in Experiment 2 .

\subsection{Methods}

\subsubsection{Participants and Stimuli}

Seventy-four participants (Age: mean $=22.06$ years, $\mathrm{SD}=8.21$ years, range = 13-39 years; Gender: 37 females, 37 males) who participated in Experiment

2 voluntarily took part also in this experiment. All 124 stimuli of Experiment 2 were also used in this experiment. The experiment was performed in the Persian language. All core variables and concepts used in this study are pro-vided in both Persian and English in Supplementary Table S1. This study was approved by the Social and Societal Ethical Committee of Tarbiat Modares University, Tehran, Iran, and all participants (and their parents if they were 
younger than 18) gave explicit informed consent prior to their participation in the experiments.

\subsubsection{Task and Procedure}

Participants viewed all 124 images one by one in a random sequence and were asked to rate the stimuli on four different five-point Likert scales to indicate how balanced, harmonious, pleasing, and interesting they found the stimuli (1, very poor; 2 , poor; 3 , fair; 4 , good; 5 , excellent), with the four scales presented one below the other, always in this order. Stimuli remained on the screen until they had clicked one of the five points on each of the four scales, after which the next stimulus was shown. The experiment lasted around 30 minutes (including voluntary breaks) with no pressure on participants to finish the rating task. The rest of the procedure and apparatus was the same as in Experiment 2.

\subsection{Results}

When compared to the altered configurations, the original artwork obtained the highest aesthetic ratings in all sets (Figs 8 and 9). However, the difference between the scores for the original artwork and the altered ones was significant (Student's $t$-test, $p<0.05$ ) in four of the six sets, namely, in Set 1R, Set 2R subsets 2 and 4, and Set 3R. In other words, altering the obliquity of the black square, translating the red square horizontally, and rotating the red square yielded the most significant effects on the aesthetics evoked by Malevich's artwork and its experimental variations. The effects were most pronounced $(p \approx 0.001)$ on the ratings of pleasingness and interestingness. Note that there are second peaks of high scores, in addition to those for the original artwork, for $0^{\circ}$ orientations of the small square, especially for balanced and harmo-nious. Replacing the small red square with a black one of the same size in sets $1 \mathrm{~B}-3 \mathrm{~B}$ did not alter these trends much. Here again the original artwork (in terms of its geometric factors) yielded the highest ratings although these trends were now no longer significant. Also the seemingly lower scores over-all for the black sets compared to the red sets, especially for pleasingness and interestingness, did not produce a statistically significant difference. Note that the secondary peaks for balanced and harmonious are as high as the origi-nal for the configurations with a small black square oriented at $0^{\circ}$; there also seems to be a second peak for harmonious for the $0^{\circ}$ skew in the large square when accompanied by a small black square.

When we studied pairwise correlations between the different aesthetic ratings, moderate to strong correlations were observed (Pearson's $r=0.6-0.9, p$ $<0.001)$. Especially strong pairwise correlations were obtained between 

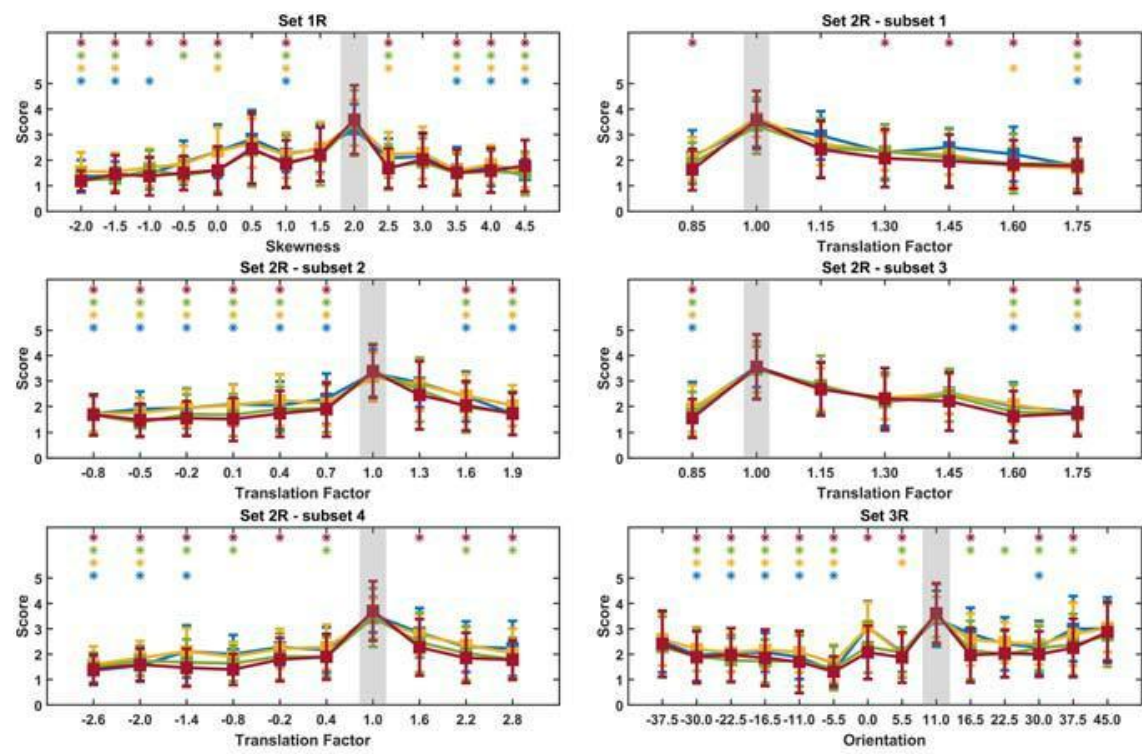

$-I$ - Batanced $-I-$ Harmonious $-\frac{I}{I}$-interesting $-I$-Plessing

Figure 8. Balanced, harmonious, interesting, and pleasing scores obtained for each stimulus in sets $1 R-3 R$ (with a small red square). Markers show the mean score obtained from the 74 participants and error bars are based on the standard deviations. The stimulus values marked by the grey zone indicate the values of the original artwork. Star marks indicate altered stimuli with statistically significant ( $p<0.05$, Student's $t$-test) lower scores than those of the original artwork. Star marks are colour-coded according to the colours used for each of the aesthetic ratings.

balanced and harmonious (Pearson's $r=0.8, p<0.001$ ), and between interesting and pleasing (Pearson's $r=0.9, p<0.001$ ).

For each stimulus, we compared the aesthetic ratings given by participants with different levels of art expertise. Interestingly, the pleasing and interesting ratings were significantly higher $(p<0.001)$ in participants with more art expertise (more experience, knowledge and interest). Balanced and harmonious ratings, however, were not significantly different between the two levels of art expertise.

\subsection{Discussion}

For the configurations with a small red square, the four aesthetic ratings were highly similar, with strong peaks for Malevich's choice in terms of skew, proximity, and orientation. In fact, the aesthetic ratings and the percentage of Yes responses (perceiving the large square as perfect) seem to be strongly related. To verify this observation, we calculated Pearson correlations, which ranged 

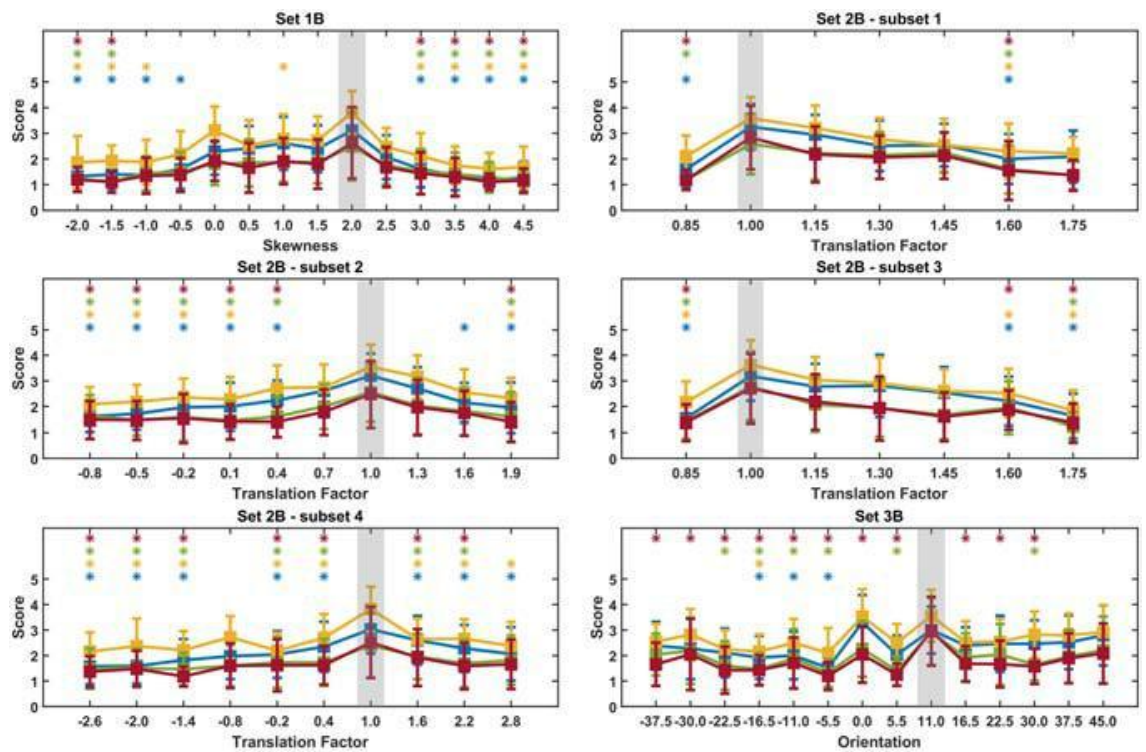

I-Balanced $-I-$ Harmonious $-I-$ interesting - - Pleasing

Figure 9. Balanced, harmonious, interesting, and pleasing scores obtained for each stimulus in Sets 1B-3B (with a small black square). Markers show the mean score obtained from the 74 participants and error bars are based on the standard deviations. The stimulus values marked by the grey zone indicate the values of the original artwork. Star marks indicate altered stimuli with statistically significant ( $p<0.05$, Student's $t$-test) lower scores than those of the original artwork. Star marks are colour-coded according to the colours used for each of the aesthetic ratings.

between 0.50 for pleasingness to 0.60 for balance (all $p<0.001$ ). Second peaks occurred for $0^{\circ}$ orientations of the small square, especially for the ratings of balanced and harmonious. For the configurations with a small black square, the four aesthetic ratings followed the same trends but the ratings of interesting and pleasing were generally lower. Here also the original geometric parameter values tended to produce the highest ratings, but there were now also strong secondary peaks for the balanced and harmonious ratings for the configurations with a small black square oriented at $0^{\circ}$ and for the harmonious rating for the $0^{\circ}$ skew in the large square. Note that these trends failed to reach statistical significance, probably due to the larger variability of the ratings in the case of the small black square configurations.

Moderate to strong correlations amongst different aesthetic ratings sug-gested that a dimensionality reduction technique [e.g., factor analysis or prin-cipal components analysis (PCA)] could be used to extract the underlying variables explaining the interrelationships among the aesthetic ratings. For each of the four different aesthetic ratings, we gathered the scores obtained 
from 74 participants for 124 stimuli. A PCA showed two orthogonal axes, one including the characteristics balanced and harmonious and the other including the characteristics interesting and pleasing. The finding of two orthogonal axes, one with more compositional terms (balanced and harmonious) and the other with more aesthetic terms (interesting and pleasing) is consistent with the idea that these two groups of ratings are processed through different pathways. Specifically, the structural terms (i.e., balanced and harmonious), are established by automatic and fast processing at the low-/mid-level visual system (Redies et al., 2015) whilst the two more aesthetic terms (i.e., interesting and pleasing) may require broader and deeper cognitive processing (Pelowski et al., 2017b).

In contrast to the previous experiments, which tapped into perception as such, the aesthetic ratings in Experiment 3 did reveal expertise effects. Interestingly, the pleasing and interesting ratings, but not the balanced and harmonious ratings, were significantly higher in participants with more art expertise. It would be interesting to speculate that the judgements of balance and harmony are more universal, while pleasingness and interestingness can be modulated more strongly by cultural background. However, because the expertise effects were not very strong, we suggest these specific findings need replication with other stimuli and samples before drawing strong conclusions.

All in all, the original configuration in Malevich's Black Square and Red Square and related configurations with the same parametric values in terms of the geometric relations between the two squares were found most interesting, pleasing, balanced and harmonious. This confirms that Malevich not only succeeded in masking the obliquity of the large square, by compensating it with a small red square in a particular position and orientation; the resulting configu-ration also generated more positive aesthetic feelings. Changing the colour of the small square from red to black made the overall configuration less interest-ing and pleasing.

\section{General Discussion}

Black Square and Red Square, a canonical abstract artwork by Kazimir Malevich, provides an interesting case study on the bridge between art his-tory/art theory and perception science/empirical aesthetics for two main rea-sons. First, it is a pioneering work in the tradition of Suprematism, which places strong emphasis on non-objective entities, dynamic forces, and the supremacy of the feelings generated by minimal compositions. Black Square and Red Square has subtle, hardly noticeable distortions, which create a mis-match between the objective, geometric properties of the shapes and their spa-tial relations, on the one hand and the subjective, perceived properties on the 
other hand. It is a minimal composition with two simple shapes, namely two squares, with different metric properties, size, colour, and orientation, but the way they are painted (intentionally inexact) and positioned implies dynamic forces between them that are supposed to induce pure feelings, and even spirituality, more so than anything from real life or representational art with its semantic and emotional associations to existing objects, scenes and events (e.g., Bimler et al., 2019). Art critics such as Milner (1996) and Luecking (2010) have discussed several perceptual and aesthetic effects of this particular composition (its shape distortions, the colour and orientation of the squares, and their spatial relations), which have never before been tested on a large sample of observers, as far as we know.

Second, Black Square and Red Square has a number of interesting proper-ties to investigate shape perception (e.g., symmetry, skew), the role of proxim-ity, colour similarity, and orientation in perceiving the exact shapes (i.e., context effects on shape perception, grouping principles), and higher-order Gestalt principles (e.g., object-centred and environment centred reference frames, localglobal relations, structural frameworks) and composition prin-ciples (e.g., order, harmony, balance, and visual rightness) that have often been proposed to play a central role in perceptual organisation and aesthetic appreciation. The minimal nature of this artwork, but the fact that it is never-theless a real artwork with high artistic importance and value, makes it an ideal case to conduct a well-controlled experimental study of the perceptual and aesthetic effects of all the factors that are supposed to play a role, with several parametric variations, a simple perceptual task (is the black square geometrically perfect or not?), and several aesthetic rating scales (five-point Likert scales of how balanced, harmonious, pleasing, and interesting the com-position is). As such, this artwork constitutes a nice compromise between the use of simple, well-controlled stimuli (still most frequent in the empirical aes-thetics literature) and the use of real paintings (more relevant to art perception and appreciation but usually less suitable for wellcontrolled experimental manipulations).

Against this background, we studied the perceptual effects of the configuration on the perception of the shape distortion of the black square in Malevich's Black Square and Red Square. In Experiment 1, we used six variations of the digitised version of the artwork (Fig. 4), to investigate how the presence and obliquity of the red square affects the perception of the geometric deviation of the black square. The results were very clear: (1) in the baseline conditions without the red square, almost all participants perceived the exact square cor-rectly, while only a minority of the participants perceived the original black square mistakenly as exact. (2) When the original distorted black square was accompanied by the small red square, it was more often seen as an exact square, reflecting a clear context effect in which the distortion of the large 
black shape is masked by the red square, as hypothesised. (3) The context effect disappeared when we replaced the red square in the original work with an exact red square, clearly indicating that the masking effect is due to the very subtle distortion in the red square. (4) When the original black square was replaced with an exact black square, there was almost no difference as a func-tion of the obliquity of the red square. (5) There was no significant difference between responses collected from less and more experienced participants, indicating that both groups perceived this geometric abstract painting simi-larly. In summary, observers perceived the original black square as an exact square in the presence of the original red square. When the red square was removed or replaced with an exact one, the obliquity of the black square was detected. The results thus showed that the simultaneous presence and obliq-uity of the red square masked the obliquity of the original black square. These findings suggest that Malevich has chosen to induce a mismatch between the objective distortion of the black shape and its subjective perception as an exact square by putting a small, subtly distorted, oriented red square below it. The fact that these effects occur in less and more experienced participants alike suggests that these principles seem to work universally.

Experiment 2 investigated in more detail how the different geometric components of the configuration formed by the black and red square affected the detectability of the obliquity of the black square in Malevich's Black Square and Red Square. Specifically, we modified a digital copy of the original art-work to create three sets of stimuli manipulating the degree of skewness of the black square's side edge, the proximity of the red square to the black square, and the orientation of the red square (Fig. 5). In addition, we also tested the role of colour similarity by repeating the same manipulations (three other sets of stimuli) with a small black square replacing the small red square. Finally, the role of art expertise was studied in more detail by including a larger sample of participants with a more refined instrument to measure art experience, art knowledge, and art interest. Again, the results were very clear: (1) we found that the distortion was most often missed in the original configuration, provid-ing strong support to the art-historical intuition that Malevich chose this par-ticular configuration to mask the obliquity of the large black square. (2) The masking effect was tuned narrowly around the original skew. (3) The masking effect depended clearly on the separation between the large black square and the small red square, especially in the vertical direction. (4) The horizontal separation between the two squares and the orientation of the red square were less important. (5) When the small red square was replaced by a small black square, the masking effects were significantly reduced, confirming the role of colour contrast as an important factor. (6) The subgroup analyses on partici-pants with different levels of art experience, art knowledge, and art interest 
revealed no statistically significant effects of art expertise on the perception of the black square in any of the six different stimulus sets.

In Experiment 3, we measured the aesthetic appreciation of Malevich's Black Square and Red Square and its alternatives (same stimuli as in Experiment 2) using four different Likert scales. Once more, the results were very clear: (1) for the configurations with a small red square, the four aesthetic ratings were highly similar, with strong peaks for Malevich's chosen values of skew, proximity, and orientation. This means that participants clearly pre-ferred the original artwork over the alternatives. They perceived the original (artistic) work as more balanced, harmonious, pleasing and interesting. (2) Secondary peaks were observed for $0^{\circ}$ orientations of the small square, espe-cially for the ratings of balance and harmony. (3) For the configurations with a small black square, the four aesthetic ratings followed the same trends but the ratings of interesting and pleasing were generally lower. Here too the origi-nal geometric parameter values tended to produce the highest ratings, with secondary peaks for balance and harmony ratings for other special configura-tions (e.g., $0^{\circ}$ skew in the large square, small black square oriented at $0^{\circ}$ ). (4) In contrast to the previous experiments, which tapped into perception as such, the aesthetic ratings in Experiment 3 did reveal expertise effects. In particular, the pleasing and interesting ratings, but not the balanced and harmonious rat-ings, were significantly higher in participants with more art expertise.

In sum, we showed that the simultaneous presence and obliquity of the red square masked the obliquity of the original black square in Malevich's Black Square and Red Square. Even slight alterations in the original configuration of the work resulted in the obliquity of the black square being noticed. The origi-nal artwork was more aesthetically preferred compared to its alternatives. These results provide strong support for the hypothesis that the artist may have intentionally set the configuration to mask the obliquity of the black square and maximise the aesthetic appeal.

Based on these findings, we would like to draw a few more general conclusions. First, we have empirically confirmed that small deviations from geomet-ric perfection, which could remain unnoticed when viewed in a single glance by untrained observers, might have intended aesthetic effects. In the case of Malevich's Black Square and Red Square, the perturbations were used to cre-ate a purely subjective (in fact, incorrect) perception of a perfect square when an imperfect large black square is put in a dynamically balanced composition with a small, tilted, subtly distorted red square. Second, the fact that this par-ticular configuration was also perceived to be most balanced, harmonious, pleasing and interesting, not only supports Malevich's successful use of his own aesthetic ideals of Suprematism; they also confirm the widely acknowl-edged role of perceptual organisation, Gestalt and composition principles underlying aesthetic appreciation. Third, the intended perceptual and aesthetic 
effects were obtained in art novices and experts alike. Although experts gave generally higher ratings of pleasingness and interestingness than novices, the novices gave similar ratings of balance and harmony, suggesting that balance and harmony might be more universal principles of aesthetic appreciation than pleasure and interest. Fourth, the fact that these findings were obtained with experimental manipulations of digital copies of a real masterpiece of abstract art demonstrates that researchers are not necessarily forced to make a choice between experimental rigour with simplistic geometric stimuli and artistic validity without experimental control. Fifth, working with abstract art, and employing a straightforward perceptual task and intuitive aesthetic ratings can produce statistically strong effects, which are theoretically meaningful for perceptual science and empirical aesthetics as well for art history, art theory and philosophy of art and aesthetics.

Of course, the specific experiments have their limitations and we would therefore like to encourage more work along these lines. We will first discuss some specific points regarding the methodology employed in the present set of experiments. First, the perceptual task was conducted for stimuli presented for a fixed duration of $2 \mathrm{~s}$, while the aesthetic ratings were given without time pressure. Although we obtained moderate correlations between the percentage of responses of a perfect square and all the aesthetic ratings (ranging from 0.5 to 0.6 ), it would be interesting to investigate whether shorter exposures would still yield the same perceptual and aesthetic effects. Second, presenting a large number of very similar stimuli to the same observers may have caused some sequential effects, including memory effects, overlearning, fatigue, and bore-dom. These were counteracted experimentally by randomising the order in which the stimuli were being presented. However, it would be worthwhile to replicate the experiments with shorter between-subjects designs. Participants in Experiment 3 (aesthetic ratings) had all participated in Experiment 2 (shape perception) as well. This could have affected the results and therefore this requires replication with a different sequential order (counterbalancing) or with independent samples of participants. Third, one could also consider an alternative procedure in which participants are asked to name the shapes they see. In this way they could freely use terms like 'probably a perfect square', 'almost a square', 'perhaps a trapezoid', or 'a quadrilateral'. As noted by McManus and Weatherby (1997), in their research on the golden section and related rectangles, the verbal labels that people apply to geometrical objects are not the same as proper geometry. Perhaps this approach could address the question whether Malevich was painting 'squares' in the verbal sense, exact squares in the geometric sense, or rather distorted squares in the geometric sense intended to be perceived as squares in the everyday sense of the word. Fourth, another suitable method for research like this would be the method of adjustment. Suppose that participants would be presented with a white 
rectangular field with a black quadrilateral and some other shapes within the field (e.g., small red squares of various sizes, orientations, relative positions). Suppose that participants would then be asked to change the shape of the quadrilateral so that it would be perceived to be a perfect square. In this way, one could easily include many variations and obtain a good range of measures for each of them (cf. Study 5 of McManus et al., 2011). Fifth, all participants were undergraduate students from a high-school in Tehran (Experiment 1, novice group) and graduate students of Tarbiat Modares University (Experiment 1, expert group; Experiments 2 and 3). We do not know to what extent the results have been influenced by their knowledge of this particular artwork or artist. In addition, it would be useful to test participants with even higher levels of art expertise (e.g., art historians or active artists) and to test the cross-cultural universality of the findings.

In addition to these more methodological limitations and extensions, there is clearly also room for further research, which should delve more deeply into the topic studied here. We may have established empirically that the relation-ships between the black and red squares mattered for the distortion of the black square to be seen or not and for the aesthetic appreciation of the overall composition, but what exactly are the relevant spatial relationships? What makes this simple configuration a good, visually right Gestalt? We have the feeling that we have only started to uncover what matters here, as some of the speculative reasons offered by art historians such as Milner (1996), for instance about the alignment of the red square's right edge with the black square's upper right-hand corner, did not seem to play a major role. This may resonate with a remark made by McManus and Kitson (1995) that compositional geom-etry is often invoked by art historians but in practice seems to have little impact on preference judgements. The more important factors probably depend less on the specific orientation of the small red square but more on the spatial sepa-ration from the big black square (especially in the vertical direction). The per-ception of the angles and lengths is probably affected by the relative sizes and orientations within and between the shapes (e.g., as we know from the hori-zontal-vertical illusion and tilt illusions such as in Witkin's rod-and-frame test), but these effects may be more categorical (right vs obtuse vs sharp angles, equal vs longer vs shorter lengths) than restricted to precise metrical properties. Undoubtedly, the reference frames established by the larger square and the overall canvas will also have an influence on the perception of the local elements such as angles and sides. Finally, the fact that the colour of the red square also mattered suggests that the relative weights of the component shapes (and perhaps even the spatial tension and dynamic forces between them) could play a role. In this context it should be noted that Malevich's spatial system was quite complicated, making use of special relationships between lengths and angles (e.g., multiples of $9^{\circ}$ ) within the shapes but also 
between the shapes of a composition, and between the different art works of an exhibition (including even the white spaces between them). Milner (1996) has identified several of these special relationships by examining the photo-graph of the 0,10 exhibition and some preserved paintings that were known to be exhibited there but it is clear that more art-historical work is still needed. For instance, why were some squares painted by Malevich real geometric squares (e.g., Black Square, 1915), why were others so distorted that they were clearly perceived as distorted (e.g., Red Square also known as Painterly Realism of a Peasant Woman in Two Dimensions, 1915), and still others, like the Black Square and Red Square studied here, subtly perturbed and combined in order to be perceived as real squares? Milner (1996) has some speculations about a particular order in the sequence of the works, but to verify these, one would have to study the chronology (and the underdrawings or different paint layers) in more detail. Note that this work would be complicated by the fact that there also forgeries on the market. On the other hand, these different ver-sions would also constitute interesting stimulus materials for a larger study with several different conditions (e.g., real squares predicted to be perceived as real squares, distorted squares predicted to be perceived as distorted squares, and distorted squares predicted to be perceived as real squares in some con-figurations but not others). Finally, Milner (1996) also has ideas about dynam-ical tensions, energy, and harmony which would be important for Malevich's Suprematism, but in their current descriptions these are a bit too vague to be tested empirically. Clearly, this line of work forms an ideal territory for more interdisciplinary research between art history, perceptual psychology, compu-tational and empirical aesthetics.

Finally, although the conclusions are in line with art-theoretical claims and a range of previous studies in perception science (on perceptual organisation and shape perception) and empirical aesthetics (on order, harmony, balance, and visual rightness), reviewed in Section 1, General Introduction, it remains the case that only one particular artwork was used (in fact, a digital copy and its experimental variations; see Hayn-Leichsenring, 2017). It is very impor-tant to replicate and extend this study to related artworks, with related princi-ples of perceptual organisation and composition, perhaps even with actual paintings in an art museum or gallery, if a real artist would produce such series of artworks with (quasi-) experimentally controlled variations. Some rigour would be lost (e.g., small differences in brush strokes, paint layer thickness, edge sharpness), probably not as many perceptual and rating trials would be feasible and the trial sequences would not be randomised, but the ecological validity and artistic relevance would be enhanced further.

In sum, based on the clear findings obtained in the present case study on Malevich's Black Square and Red Square, we can only encourage more work along these lines. 


\section{Notes}

1. From a purely mathematical point of view, the shape of the Black Square should not be described as a square but rather as a right trapezium, while the Red Square is probably best described as a quadrilateral. In everyday language, imperfect squares are often still described as squares because they are perceived as squares. However, here we ask observers for a range of 'squares' (two perfect ones and the rest distorted ones) to indicate whether they are real, mathematically exact squares or not. In this sense, there seem to be good arguments to use the exact mathematical terms and use 'square' for the distorted ones only when referring to the title of the art work. However, after some deliberation, we argued against this for reasons of simplicity and readability. Because the whole focus of the study is on perception, not reality, and Malevich's Suprematism empha-sises the non-objectivity of paintings such as Black Square and Red Square, we decided to write about black and red squares, even when they are imperfect. Only in statements where it is important to distinguish between a geometrically exact square and a deformed one, we use the term 'exact square', which we believe is sufficient for readers to follow what we did.

2. When trying to measure the geometrical aspects of the paintings, one is confronted with the problem that different images and sources could yield slightly different measurements. For this reason, we decided to rely on an art-historical authority, who focused on the geometry of Malevich's abstract art works, based on precise drawings of rastered scans. Note also that Malevich used vershok, a Russian Imperial measure of length (around $4.445 \mathrm{~cm}$ ), which often masks the regularities in Malevich's spa-tial system when formulated in metric units.

3. In all three experiments, we compared the results between female and male participants and found no statistically significant differences.

\section{Supplementary Material}

Supplementary material is available online at: https://doi.org/10.6084/m9.figshare.16692043

\section{References}

Amirshahi, S. A., Hayn-Leichsenring, G. U., Denzler, J., and Redies, C. (2014). Evaluating the rule of thirds in photographs and paintings, Art Percept. 2, 163-182. doi: 10.1163/ 22134913-00002024. 
Arnheim, R. (1971). Entropy and Art: An Essay on Disorder and Order. University of California Press, Berkeley, CA, USA.

Arnheim, R. (1982). The Power of the Center: A Study of Composition in the Visual Arts. University of California Press, Berkeley, CA, USA.

Arnheim, R. (2004). Art and Visual Perception: A Psychology of the Creative Eye (50th anniversary printing, revised edition). University of California Press, Berkeley, CA, USA. (Original published 1954)

Bimler, D. L., Snellock, M., \& Paramei, G. V. (2019). Art expertise in construing meaning of representational and abstract artworks, Acta Psychol. 192, 11-22. doi: 10.1016/j.actpsy. 2018.10.012.

Boselie, F. (1992). The golden section has no special aesthetic attractivity, Empir. Stud. Arts 10, 1-18. doi: 10.2190/QB14-NK7B-ARYT-W5QT.

Brielmann, A. A., \& Pelli, D. G. (2018). Aesthetics, Curr. Biol. 28, R859-R863. doi: 10.1016/j. cub.2018.06.004.

Bruno, N. (2020). Thirty-six views of X: variations on a theme reveal individual artists' approaches to composition, Art Percept. 8, 158-174. doi: 10.1163/22134913-bja10003.

Carbon, C.-C. (2020). Ecological art experience: how we can gain experimental control while preserving ecologically valid settings and contexts, Front. Psychol. 11, 800. doi: 10.3389/ fpsyg.2020.00800.

Chamberlain, R., \& Wagemans, J. (2015). Visual arts training is linked to flexible attention to local and global levels of visual stimuli, Acta Psychol. 161, 185-197. doi: 10.1016/j. actpsy.2015.08.012.

Claessens, P. M., \& Wagemans, J. (2005). Perceptual grouping in Gabor lattices: Proximity and alignment, Percept. Psychophys. 67, 1446-1459. doi: 10.3758/BF03193649.

Claessens, P. M., \& Wagemans, J. (2008). A Bayesian framework for cue integration in multistable grouping: Proximity, collinearity, and orientation priors in zigzag lattices, J. Vis. 8, 33.1-33.23. doi: 10.1167/8.7.33.

Corrada, M. (1997). "Kazimir Malevich and the art of geometry”, John Milner (Book Review), Structurist 37, 109.

Crone, R., \& Moos, D. (1991). Kazimir Malevich: the Climax of Disclosure. Reaktion Books Ltd, London, UK.

Douglas, C. (1998). Kazimir Malevich and the art of geometry, by John Milner (Book Review). Slavic Rev. 57, 233-234. doi: 10.2307/2502105.

Fillinger, M. G., \& Hübner, R. (2020). Relations between balance, prototypicality, and aesthetic appreciation for Japanese calligraphy, Empir. Stud. Arts 38, 172-190. doi: 10.1177/ 0276237418805656.

Fingerhut, J., \& Prinz, J. J. (2018). Wonder, appreciation, and the value of art, Prog. Brain Res. 237, 107-128. doi: 10.1016/bs.pbr.2018.03.004.

Fol Leymarie, F., \& Aparajeya, P. (2017). Medialness and the perception of visual art, Art Percept. 5, 169-232. doi: 10.1163/22134913-00002064.

Galenson, D. W. (2008). Two paths to abstract art: Kandinsky and Malevich, Russ. Hist. 35, 236-250. doi: 10.1163/187633108X00283.

Gershoni, S., \& Hochstein, S. (2011). Measuring pictorial balance perception at first glance using Japanese calligraphy, $i$-Perception 2, 508-527. doi: 10.1068/i0472aap. 
Golding, J. (2000). Paths to the Absolute: Mondrian, Malevich, Kandinsky, Pollock, Rothko, and Still. Princeton University Press, Princeton, NJ, USA.

Graf, L. K. M., \& Landwehr, J. R. (2017). Aesthetic pleasure versus aesthetic interest: the two routes to aesthetic liking, Front. Psychol. 8, 15. doi: 10.3389/fpsyg.2017.00015.

Green, C. D. (1995). All that glitters: a review of psychological research on the aesthetics of the golden section, Perception 24, 937-968. doi: 10.1068/p240937.

Guidi, S., \& Palmer, S. E. (2015). The internal structure of a rectangular frame: Position, orientation, and direction, Art Percept. 3, 19-52. doi: 10.1163/22134913-00002013.

Harrison, C., \& Wood, P. (2003). Art in Theory, 1900-2000: an Anthology of Changing Ideas. Blackwell, Oxford, UK.

Hayn-Leichsenring, G. U. (2017). The ambiguity of artworks - a guideline for empirical aesthetics research with artworks as stimuli, Front. Psychol. 8, 1857. doi: 10.3389/ fpsyg.2017.01857.

Hübner, R., \& Fillinger, M. G. (2016). Comparison of objective measures for predicting perceptual balance and visual aesthetic preference, Front. Psychol. 7, 335. doi: 10.3389/fpsyg. 2016.00335.

Hübner, R., \& Fillinger, M. G. (2019). Perceptual balance, stability, and aesthetic appreciation: their relations depend on the picture type, i-Perception 10, 2041669519856040. doi: $10.1177 / 2041669519856040$.

Jacobsen, T. (2006). Bridging the arts and sciences: A framework for the psychology of aesthetics, Leonardo 39, 155-162. doi: 10.1162/leon.2006.39.2.155.

Jacobsen, T., \& Höfel, L. (2002). Aesthetic judgments of novel graphic patterns: analyses of individual judgments, Percept. Mot. Skills 95, 755-766. doi: 10.2466/pms.2002.95.3.755.

Kochman, A. (1999). John Milner, Kazimir Malevich and the Art of Geometry (Book Review), J. Ukr. Stud. 24, 118-119.

Koenderink, J. J. (2015a). Perceptual organization in visual art, in: Oxford Handbook of Perceptual Organization, J. Wagemans (Ed.), pp. 886-916, Oxford University Press, Oxford, U.K. doi: 10.1093/oxfordhb/9780199686858.013.045.

Koenderink, J. J. (2015b). Parts and wholes in pictorial art, Art Percept. 3, 303-317. doi: 10.1163/22134913-00002035.

Koenderink, J. J., van Doorn, A. J., \& Gegenfurtner, K. (2017). Compositorial 'weight' \& 'luminance', Art Percept. 5, 299-311. doi: 10.1163/22134913-00002067.

Kubovy, M., Holcombe, A. O., \& Wagemans, J. (1998). On the lawfulness of grouping by proximity, Cogn. Psychol. 35, 71-98. doi: 10.1006/cogp.1997.0673.

Kubovy, M., \& van den Berg, M. (2008). The whole is equal to the sum of its parts: a probabilistic model of grouping by proximity and similarity in regular patterns, Psychol. Rev. 115, 131-154. doi: 10.1037/0033-295X.115.1.131.

Kubovy, M., \& Wagemans, J. (1995). Grouping by proximity and multistability in dot lattices: a quantitative Gestalt theory, Psychol. Sci. 6, 225-234. doi:10.1111/j.1467-9280.1995. tb00597.x.

Latto, R., Brain, D., \& Kelly, B. (2000). An oblique effect in aesthetics: homage to Mondrian (1872-1944), Perception 29, 981-987. doi: 10.1068/p2352.

Leder, H., Gerger, G., Brieber, D., \& Schwarz, N. (2014). What makes an art expert? Emotion and evaluation in art appreciation, Cogn. Emot. 28, 1137-1147. doi: 10.1080/ 02699931.2013.870132. 
Leder, H., Tinio, P. P. L., Brieber, D., Kröner, T., Jacobsen, T., \& Rosenberg, R. (2019). Symmetry is not a universal law of beauty, Empir. Stud. Arts 37, 104-114. doi: 10.1177/ 0276237418777941.

Letsch, P., \& Hayn-Leichsenring, G. U. (2020). The composition of abstract images: Differences between artists and laypersons, Psychol. Aesthet. Creat. Arts 14, 186-196. doi: $10.1037 /$ aca0000209.

Locher, P. J. (2003). An empirical investigation of the visual rightness theory of picture perception, Acta Psychol. 114, 147-164. doi: 10.1016/j.actpsy.2003.07.001.

Locher, P., Gray, S., \& Nodine, C. (1996). The structural framework of pictorial balance, Perception 25, 1419-1436. doi: 10.1068/p251419.

Locher, P., \& Nagy, Y. (1996). Vision spontaneously establishes the percept of pictorial balance, Empir. Stud. Arts 14, 17-31. doi: 10.2190/X8U3-CTQ6-A7J1-8JQ8.

Locher, P. J., Stappers, P. J., \& Overbeeke, K. (1998). The role of balance as an organizing design principle underlying adults' compositional strategies for creating visual displays, Acta Psychol. 99, 141-161. doi: 10.1016/S0001-6918(98)00008-0.

Locher, P. J., Stappers, P. J., \& Overbeeke, K. (1999a). An empirical evaluation of the visual rightness theory of pictorial composition, Acta Psychol. 103, 261-280. doi: 10.1016/ S00016918(99)00044-X.

Locher, P., Smith, L., \& Smith, J. (1999b). Original paintings versus slide and computer reproductions: a comparison of viewer responses, Empir. Stud. Arts 17, 121-129. doi: 10.2190/ R1WN-TAF2-376D-EFUH.

Locher, P., Cornelis, E., Wagemans, J., \& Stappers, P. J. (2001a). Artists' use of compositional balance for creating visual displays, Empir. Stud. Arts 19, 213-227. doi: 10.2190/ EKMDYMN5-NJUG-34BK.

Locher, P. J., Smith, J. K., \& Smith, L. F. (2001b). The influence of presentation format and viewer training in the visual arts on the perception of pictorial and aesthetic qualities of paintings, Perception 30, 449-465. doi: 10.1068/p3008.

Locher, P. J., Overbeeke, K., \& Stappers, P. J. (2005). Spatial balance of color triads in the abstract art of Piet Mondrian, Perception 34, 169-189. doi: 10.1068/p5033.

Luecking, S. (2010). A man and his square: Kazimir Malevich and the visualization of the fourth dimension, J. Math. Arts 4, 87-100. doi: 10.1080/17513471003744395.

Marković, S. (2012). Components of aesthetic experience: aesthetic fascination, aesthetic appraisal, and aesthetic emotion, i-Perception 3, 1-17. doi: 10.1068/i0450aap.

McManus, I. C. (2005). Symmetry and asymmetry in aesthetics and the arts, Eur. Rev. 13, $157-$ 180. doi: $10.1017 /$ S1062798705000736.

McManus, I. C., Cheema, B., \& Stoker, J. (1993). The aesthetics of composition: a study of Mondrian, Empir. Stud. Arts 11, 83-94. doi: 10.2190/HXR4-VU9A-P5D9-BPQQ.

McManus, I. C., Edmondson, D., \& Rodger, J. (1985). Balance in pictures, Br. J. Psychol. 76, 311-324. doi: 10.1111/j.2044-8295.1985.tb01955.x.

McManus, I. C., \& Kitson, C. M. (1995). Compositional geometry in pictures, Empir. Stud. Arts 13, 73-94. doi: 10.2190/66DJ-GVVJ-A33U-X7AT.

McManus, I. C., Stöver, K., \& Kim, D. (2011). Arnheim's Gestalt theory of visual balance: examining the compositional structure of art photographs and abstract images, $i$-Perception 2, 615-647. doi: 10.1068/i0445aap. 
McManus, I. C., \& Weatherby, P. (1997). The golden section and the aesthetics of form and composition: a cognitive model, Empir. Stud. Arts 15, 209-232. doi: 10.2190/WWCRVWHV-2Y2W-91EE.

Menzel, C., Kovács, G., Amado, C., Hayn-Leichsenring, G. U., \& Redies, C. (2018). Visual mismatch negativity indicates automatic, task-independent detection of artistic image composition in abstract artworks, Biol. Psychol. 136, 76-86. doi: 10.1016/j.biopsycho. 2018.05.005.

Milner, J. (1996). Kazimir Malevich and the Art of Geometry. Yale University Press, New Haven, CT, USA.

Muth, C., Hesslinger, V. M., \& Carbon, C. C. (2015). The appeal of challenge in the perception of art: How ambiguity, solvability of ambiguity, and the opportunity for insight affect appreciation, Psychol. Aesthet. Creat. Arts 9, 206-216. doi: 10.1037/a0038814.

Muth, C., Westphal-Fitch, G., \& Carbon, C.-C. (2021). Seeking (dis)order: Ordering appeals but slight disorder and complex order trigger interest, Psychol. Aesthet. Creat. Arts 15, 439457. doi: 10.1037/aca0000284.

Palmer, S. E., Schloss, K. B., \& Sammartino, J. (2013). Visual aesthetics and human preference, Annu. Rev. Psychol. 64, 77-107. doi: 10.1146/annurev-psych-120710-100504.

Pelowski, M., Forster, M., Tinio, P. P. L., Scholl, M., \& Leder, H. (2017a). Beyond the lab: An examination of key factors influencing interaction with 'real' and museum-based art, Psychol. Aesthet. Creat. Arts 11, 245-264. doi: 10.1037/aca0000141.

Pelowski, M., Markey, P. S., Forster, M., Gerger, G., \& Leder, H. (2017b). Move me, astonish me... delight my eyes and brain: The Vienna Integrated Model of top-down and bottom-up processes in Art Perception (VIMAP) and corresponding affective, evaluative, and neuro-physiological correlates, Phys. Life Rev. 21, 80-125. doi: 10.1016/j.plrev.2017.02.003.

Pinna, B. (2010). New Gestalt principles of perceptual organization: An extension from grouping to shape and meaning, Gestalt Theory 32, 11-78.

Pinna, B. (2011). What is the meaning of shape? Gestalt Theory 33, 383-422.

Pinna, B., \& Deiana, K. (2015). The syntax organization of shape and color and the laws of coloration in vision, art and biology, Art Percept. 3, 319-345. doi: 10.1163/2213491300002034.

Redies, C. (2015). Combining universal beauty and cultural context in a unifying model of visual aesthetic experience, Front. Hum. Neurosci. 9, 218. doi: 10.3389/fnhum.2015.00218.

Redies, C., Brachmann, A., \& Hayn-Leichsenring, G. U. (2015). Changes of statistical properties during the creation of graphic artworks, Art Percept. 3, 93-116. doi: 10.1163/ 22134913-00002017.

Rosenberg, R., \& Klein, C. (2015). The moving eye of the beholder: Eye tracking and the perception of paintings, in: Art, Aesthetics and the Brain, J. P. Huston, M. Nadal, F. Mora, L. F. Agnati, \& C. J. Cela-Conde (Eds), pp. 79-108, Oxford University Press, Oxford, UK. doi: 10.1093/acprof:oso/9780199670000.003.0005.

Schwabe, K., Menzel, C., Mullin, C., Wagemans, J., \& Redies, C. (2018). Gist perception of image composition in abstract artworks, i-Perception 9, 2041669518780797. doi: 10.1177/ 2041669518780797.

Serrao, F. (2019). Enjoying art: the experience of beauty from understanding regularities, Art Percept. 7, 137-175. doi: 10.1163/22134913-20191106. 
Shimamura, A. P., \& Palmer, S. E. (2012). Aesthetic Science: Connecting Minds, Brains, and Experience. Oxford University Press, New York, NY, USA.

Silvia, P. J. (2007). Knowledge-based assessment of expertise in the arts: Exploring aesthetic fluency, Psychol. Aesthet. Creat. Arts 1, 247-249. doi: 10.1037/1931-3896.1.4.247.

Silvia, P. J. (2009). Looking past pleasure: Anger, confusion, disgust, pride, surprise, and other unusual aesthetic emotions, Psychol. Aesthet. Creat. Arts 3, 48-51. doi: 10.1037/a0014632.

Smith, L. F., \& Smith, J. K. (2006). The nature and growth of aesthetic fluency, in: Foundations and Frontiers in Aesthetics. New directions in aesthetics, creativity and the arts, P. Locher, C. Martindale, \& L. Dorfman (Eds), pp. 47-58. Baywood Publishing Company, Amityville, NY, USA.

Specker, E., Forster, M., Brinkmann, H., Boddy, J., Pelowski, M., Rosenberg, R., \& Leder, H. (2020). The Vienna Art Interest and Art Knowledge Questionnaire (VAIAK): A unified and validated measure of art interest and art knowledge, Psychol. Aesthet. Creat. Arts 14, 172185. doi: 10.1037/aca0000205.

Spehar, B., \& van Tonder, G. J. (2017). Koffka's aesthetic Gestalt, Leonardo 50, 5357. doi: 10.1162/LEON_a_01020.

Tao, W., Liu, Y., \& Zhang, K. (2014). Automatically generating abstract paintings in Malevich Style, in: 2014 IEEE/ACIS 13th International Conference on Computer and Information Science (ICIS) pp. 201-205. doi: 10.1109/ICIS.2014.6912134.

Tinio, P. P. L., \& Smith, J. K. (2014). The Cambridge Handbook of the Psychology of Aesthetics and the Arts. Cambridge University Press, Cambridge, UK.

Van de Cruys, S., \& Wagemans, J. (2011a). Putting reward in art: a tentative prediction error account of visual art, i-Perception 2, 1035-1062. doi: 10.1068/i0466aap.

Van de Cruys, S., \& Wagemans, J. (2011b). Gestalts as predictions: Some reflections and an application to art, Gestalt Theory 33, 325-344.

Van Geert, E., \& Wagemans, J. (2020). Order, complexity, and aesthetic appreciation, Psychol. Aesthet. Creat. Arts 14, 135-154. doi: 10.1037/aca0000224.

Van Gool, L. J., Moons, T., Pauwels, E., \& Wagemans, J. (1994). Invariance from the Euclidean geometer's perspective, Perception 23, 547-561. doi: 10.1068/p230547.

van Tonder, G., \& Spehar, B. (2013). The aesthetic appeal of visual qualities, in: Handbook of Experimental Phenomenology: Visual Perception of Shape, Space and Appearance, L. Albertazzi (Ed.), pp. 395-414. doi: 10/10.1002/9781118329016.ch16.

Wagemans, J. (1993). Skewed symmetry: A nonaccidental property used to perceive visual forms, J. Exp. Psychol. Hum. Percept. Perform. 19, 364-380. doi: 10.1037/00961523.19.2.364.

Wagemans, J. (1997). Characteristics and models of human symmetry detection, Trends Cogn. Sci. 1, 346-352. doi: 10.1016/S1364-6613(97)01105-4.

Wagemans, J. (2015a). Historical and conceptual background: Gestalt theory, in: The Oxford Handbook of Perceptual Organization, J. Wagemans (Ed.), pp. 3-20. Oxford University Press, Oxford, UK.

Wagemans, J. (2015b). The Oxford Handbook of Perceptual Organization, Oxford University Press, Oxford, UK.

Wagemans, J. (2017). Adding Gestalt to the picture. Comment on "Move me, astonish me... delight my eyes and brain: The Vienna Integrated Model of top-down and bottom-up processes in Art Perception (VIMAP) and corresponding affective, evaluative, and 
neurophysiological correlates" by Matthew Pelowski et al. Phys. Life Rev. 21, 155-158. doi: 10.1016/j.plrev.2017.06.009.

Wagemans, J. (2018). Perceptual organization, in: Stevens' Handbook of Experimental Psychology and Cognitive Neuroscience: Vol. 2. Sensation, Perception \& Attention, J. T. Wixted \& J. Serences (Eds), pp. 803-872. John Wiley \& Sons, Hoboken, NJ, USA. doi: 10.1002/9781119170174.epcn218.

Wagemans, J., Van Gool, L., Swinnen, V., \& Van Horebeek, J. (1993). Higher-order structure in regularity detection, Vision Res. 33, 1067-1088. doi: 10.1016/0042-6989(93)90241-N.

Wagemans, J., Vanden Bossche, P., Segers, N., \& d'Ydewalle, G. (1994). An affine group model and the perception of orthographically projected planar random polygons, J. Math. Psychol. 38, 59-72. doi: 10.1006/jmps.1994.1003.

Wagemans, J., Lamote, C., \& Van Gool, L. (1997). Shape equivalence under perspective and projective transformations, Psychon. Bull. Rev. 4, 248-253. doi: 10.3758/BF03209401.

Wagemans, J., Elder, J. H., Kubovy, M., Palmer, S. E., Peterson, M. A., Singh, M., \& von der Heydt, R. (2012a). A century of Gestalt psychology in visual perception: I. Perceptual grouping and figure-ground organization, Psychol. Bull. 138, 1172-1217. doi: 10.1037/ a0029333.

Wagemans, J., Feldman, J., Gepshtein, S., Kimchi, R., Pomerantz, J. R., van der Helm, P., \& van Leeuwen, C. (2012b). A century of Gestalt psychology in visual perception: II. Conceptual and theoretical foundations, Psychol. Bull. 138, 1218-1252. doi: 10.1037/a0029334.

Weichselbaum, H., Leder, H., \& Ansorge, U. (2018). Implicit and explicit evaluation of visual symmetry as a function of art expertise, i-Perception 9, 2041669518761464. doi: $10.1177 / 2041669518761464$

Westphal-Fitch, G., Huber, L., Gómez, J. C., \& Fitch, W. T. (2012). Production and perception rules underlying visual patterns: effects of symmetry and hierarchy, Philos. Trans. R. Soc. Lond. B Biol. Sci. 367, 2007-2022. doi: 10.1098/rstb.2012.0098.

Winston, A. S., \& Cupchik, G. C. (1992). The evaluation of high art and popular art by naive and experienced viewers. Vis. Arts Res. 18, 1-14. http://www.jstor.org/stable/20715763.

Wright, D., \& Bertamini, M. (2015). Aesthetic judgements of abstract dynamic configurations, Art Percept. 3, 283-301. doi: 10.1163/22134913-00002037.

Zhu, L. (2016). Outline of a theory of visual tension, Art Percept. 4, 127-144. doi: 10.1163/22134913-00002048. 